Homology, Homotopy and Applications, vol.11(2), 2009, pp.195-222

\title{
ON STRUCTURE SETS OF MANIFOLD PAIRS
}

\author{
MATIJA CENCELJ, YURI V. MURANOV AND DUŠAN REPOVŠ
}

(communicated by Maria Cristina Pedicchio)

Abstract

In this paper we systematically describe relations between various structure sets which arise naturally for pairs of compact topological manifolds with boundary. Our consideration is based on a deep analogy between the case of a compact manifold with boundary and the case of a closed manifold pair. This approach also gives a possibility to construct the obstruction groups for natural maps of various structure sets and to investigate their properties.

\section{Introduction}

Let

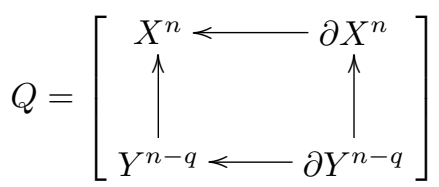

be a pair of compact topological manifolds with boundary such that $\partial Y \subset \partial X$ and $Y \subset X$ are locally flat submanifolds with given structures of the normal bundles (cf. $[\mathbf{8}]$ and $[\mathbf{9}]$ ).

The pair of manifolds in (1) is a special case of a stratified manifold (cf. [9] and $[\mathbf{1 1}]$ ), and a complete description of the relations between various structure sets in this situation is very helpful for the investigation of the general situation of a stratified manifold. In this paper we systematically describe relations between different structure sets which arise naturally in this case. We shall work in the category of topological manifolds and assume that the dimension of all manifolds is greater than or equal to 5 . We shall use surgery theory for the case of simple homotopy equivalence (cf. $[\mathbf{8}, \mathbf{9}]$ and $[\mathbf{1 0}]$ ), and we shall tacitly assume that all obstruction groups are decorated with " $s$ ". We shall use the functoriality of the basic maps in surgery theory and the algebraic surgery theory of Ranicki (cf. $[\mathbf{1}, \mathbf{3}, \mathbf{6}, \mathbf{8}]$ and $[\mathbf{9}]$ ).

In Section 2 we define classical structure sets for a manifold with boundary $(X, \partial X)$ and we describe the natural relations among them. In Section 3 we define structure sets for a closed manifold pair $\left(X^{n}, Y^{n-q}\right)$ and we describe the natural relations

Received January 18, 2009, revised August 10, 2009; published on November 24, 2009.

2000 Mathematics Subject Classification: 57R67, 19J25, 55T99, 58A35, 18 F25.

Key words and phrases: surgery on manifolds, surgery on manifold pairs, surgery obstruction groups, splitting obstruction groups, surgery exact sequence, structure sets, normal invariants.

This article is available at http://intlpress.com/HHA/v11/n2/a10

Copyright (c) 2009, International Press. Permission to copy for private use granted. 
among them. We point out a deep analogy between the case $(X, \partial X)$ considered in Section 2 and the case of a closed manifold pair $(X, Y)$ considered in Section 3.

In Section 4 we present the necessary material on the realization of the structure sets introduced above, on the spectrum level $([\mathbf{1}, \mathbf{2}, \mathbf{4}, \mathbf{5}, \mathbf{6}, \mathbf{8}]$, and $[\mathbf{9}])$. From this realization and the results of the previous sections, we derive the well-known basic diagrams of exact sequences for the structure sets (cf. $[\mathbf{2}, \mathbf{4}, \mathbf{5}, \mathbf{8}, \mathbf{9}]$ and $[\mathbf{1 0}])$. In this section we also describe the analogy between $(X, \partial X)$ and a closed manifold pair $(X, Y)$ on the level of the natural maps between the structure sets. This approach gives a possibility to better understand the geometric properties of natural maps in surgery theory.

In Section 5 we extend our consideration to the case of a compact manifold pair with boundary. The results of this section are known to the experts, but not all of them have been published until now. Our approach gives an opportunity to systematically describe the structure sets of such pairs and the natural maps between them. The naturality of this approach also provides realization of structure sets and the natural maps between them on the spectrum level. In this way it is possible to describe all structure sets for a closed manifold pair with boundary and describe relations among them, other structure sets, and the surgery obstruction groups.

In Section 6 we point out some possible applications of our approach to the description of various obstruction groups which arise naturally when we consider the natural maps between structure sets.

\section{Acknowledgements}

This research was supported by the Slovenian Research Agency grants P1-02920101 and J1-9643-0101.

\section{The structure sets of $(X, \partial X)$}

Let $X^{n}$ be a closed $n$-dimensional manifold. An $s$-triangulation of $X$ is a simple homotopy equivalence $f: M \rightarrow X$, where $M^{n}$ is a closed $n$-dimensional topological manifold (cf. $[\mathbf{8}, \mathbf{9}]$ and $[\mathbf{1 0}]$ ). Two $s$-triangulations, $f_{i}: M_{i} \rightarrow X$, are concordant (cf. [9]) if there exists a simple homotopy equivalence of triads

$$
\left(F ; f_{0}, f_{1}\right):\left(W ; M_{0}, M_{1}\right) \rightarrow(X \times I ; X \times\{0\}, X \times\{1\}),
$$

where $W$ is a compact $(n+1)$-dimensional manifold with the boundary

$$
\partial W=M_{0} \cup M_{1} .
$$

The set of concordance classes is denoted by $\mathcal{S}(X)$.

A $t$-triangulation of $X$ is a topological normal map $(f, b): M \rightarrow X$, where $M^{n}$ is a closed $n$-dimensional topological manifold (cf. $[\mathbf{8}]$ and $[\mathbf{9}]$ ). Two $t$-triangulations are concordant (cf. [9]) if there exists a topological normal map of triads

$$
\left((F, C) ;\left(f_{0}, b_{0}\right),\left(f_{1}, b_{1}\right)\right):\left(W ; M_{0}, M_{1}\right) \rightarrow(X \times I ; X \times\{0\}, X \times\{1\}) .
$$

The set of concordance classes of $t$-triangulations of $X$ is denoted by $\mathcal{T}(X)$. 
For $n \geqslant 5$ these sets fit into the surgery exact sequence (cf. $[\mathbf{8}, \mathbf{9}]$ and $[\mathbf{1 0}]$ )

$$
\cdots \rightarrow L_{n+1}\left(\pi_{1}(X)\right) \rightarrow \mathcal{S}(X) \rightarrow \mathcal{T}(X) \rightarrow L_{n}\left(\pi_{1}(X)\right) \rightarrow \cdots,
$$

where $L_{*}\left(\pi_{1}(X)\right)$ are the surgery obstruction groups.

Thus for a closed manifold $X$ we have two types of structures, which are denoted by $\mathcal{S}$ and $\mathcal{T}$. We can write down the following diagram for these structures:

$$
\mathcal{S} \longrightarrow \mathcal{T} \text {. }
$$

We can interpret the arrow in (3) as a relation of partial order, and we can say that the structure $\mathcal{S}$ is 'stronger' than the structure $\mathcal{T}$.

Diagram (3) means that, for any manifold $X$, a simple homotopy equivalence of $n$-manifolds, $f: M \rightarrow X$, which represents an element of the structure set $\mathcal{S}(X)$, correctly defines a structure in $\mathcal{T}(X)$ (cf. [10]), that is the map $\mathcal{S}(X) \rightarrow \mathcal{T}(X)$ in $(2)$. The map of structures in (3) is the map of 'weakening of the structure'.

Let $\left(X^{n}, \partial X^{n}\right)$ be a compact manifold with boundary. In this case, there are the following five structures on $(X, \partial X)$ :

$$
\mathcal{T} \mathcal{T}, \quad \mathcal{T} \mathcal{S}, \quad \mathcal{T} \mathcal{D}, \quad \mathcal{S S} \text { and } \mathcal{S D}
$$

The first two structures on $(X, \partial X)$ in (4) correspond to $\mathcal{T}$-structures on $X$ whose restrictions to the boundary give the structures $\mathcal{T}$ and $\mathcal{S}$. The third structure corresponds to $\mathcal{T}$-structures on $X$ whose restriction to $\partial X$ is a homeomorphism and the equivalence relation is considered rel the boundary. The last two structures on $(X, \partial X)$ in (4) are considered similarly.

For a compact manifold with boundary $\left(X^{n}, \partial X\right)$, we recall the definition of the structure sets which correspond to the structures from (4) (cf. $[\mathbf{4 , 9}]$ and $[\mathbf{1 0}])$.

$(\mathcal{T} \mathcal{T})$. This is the relative case in the sense of $[\mathbf{9}]$ and $[\mathbf{1 0}]$. Let

$$
((f, b),(\partial f, \partial b)):(M, \partial M) \rightarrow(X, \partial X)
$$

be a $t$-triangulation of the pair $(X, \partial X)$. Two $t$-triangulations,

$$
\left(\left(f_{i}, b_{i}\right),\left(\partial f_{i}, \partial b_{i}\right)\right):\left(M_{i}, \partial M_{i}\right) \rightarrow(X, \partial X), i=0,1,
$$

are concordant if there exists a $t$-triangulation of the 4-ad

$$
\begin{aligned}
\left((F, B) ;(g, c),\left(f_{0}, b_{0}\right),\left(f_{1}, b_{1}\right)\right):(W ; V, & \left.M_{0}, M_{1}\right) \\
& \rightarrow(X \times I ; \partial X \times I, X \times\{0\}, X \times\{1\})
\end{aligned}
$$

with $\partial V=\partial M_{0} \cup \partial M_{1}$. The set of concordance classes is denoted by $\mathcal{T}(X, \partial X)$.

$(\mathcal{T S})$. This is the mixed structure on a manifold with boundary in the sense of $[4]$ and [10]. Let $f:(M, \partial M) \rightarrow(X, \partial X)$ be a $t$-triangulation of $(X, \partial X)$ such that the restriction $\left.f\right|_{\partial M}: \partial M \rightarrow \partial X$ is an $s$-triangulation. Two such maps, $f_{i}:\left(M_{i}, \partial M_{i}\right) \rightarrow$ $(X, \partial X), i=0,1$, are concordant if there exists a t-triangulation (5) of the 4-ad such that $V=F^{-1}(\partial X \times I)$ and the restriction $\left.F\right|_{V}$ is an $s$-triangulation

$$
\left(\left.F\right|_{V} ;\left.F\right|_{\partial M_{0}},\left.F\right|_{\partial M_{1}}\right):\left(V ; \partial M_{0}, \partial M_{1}\right) \rightarrow(\partial X \times I ; \partial X \times\{0\}, \partial X \times\{1\})
$$

of the triad $(\partial X \times I ; \partial X \times\{0\}, \partial X \times\{1\})$. The set of equivalence classes of such maps is denoted by $\mathcal{T} \mathcal{S}(X, \partial X)($ cf. [4]). 
$(\mathcal{T D})$. This is the rel $_{\partial}$-case in the sense of $[\mathbf{9}]$ and $[\mathbf{1 0}]$ (cf. also $[\mathbf{4}]$ and $\left.[\mathbf{5}]\right) . \mathrm{A}$ $t_{\partial}$-triangulation of $(X, \partial X)$ is a $t$-triangulation

$$
((f, b),(\partial f, \partial b)):(M, \partial M) \rightarrow(X, \partial X)
$$

whose restriction $\partial f$ to the boundary is a homeomorphism $\partial M \rightarrow \partial X$. Two $t_{\partial^{-}}$ triangulations,

$$
\left(\left(f_{i}, b_{i}\right),\left(\partial f_{i}, \partial b_{i}\right)\right):\left(M_{i}, \partial M_{i}\right) \rightarrow(X, \partial X), i=0,1
$$

are concordant if there exists a $t$-triangulation (5) of the 4-ad with the condition

$$
V=\partial M_{0} \times I, \quad(g, c)=\partial f_{0} \times I: V \rightarrow \partial X \times I .
$$

The set of concordance classes is denoted by $\mathcal{T}^{\partial}(X, \partial X)$.

$(\mathcal{S S})$. This is the relative case of $s$-triangulations in the sense of $[\mathbf{9}]$ and $[\mathbf{1 0}]$. Let

$$
(f, \partial f):(M, \partial M) \rightarrow(X, \partial X)
$$

be an $s$-triangulation of the pair $(X, \partial X)$. Two $s$-triangulations,

$$
\left(f_{i}, \partial f_{i}\right):\left(M_{i}, \partial M_{i}\right) \rightarrow(X, \partial X), i=0,1,
$$

are concordant if there exists a simple homotopy equivalence of 4-ads

$$
\left(\left(F ; g, f_{0}, f_{1}\right)\right):\left(W ; V, M_{0}, M_{1}\right) \rightarrow(X \times I ; \partial X \times I, X \times\{0\}, X \times\{1\})
$$

with

$$
\partial V=\partial M_{0} \cup \partial M_{1}
$$

The set of concordance classes is denoted by $\mathcal{S}(X, \partial X)$.

$(\mathcal{S D})$. This is the case of $s_{\partial}$-triangulation in the sense of $[\mathbf{4}, \mathbf{5}]$, and $[\mathbf{9}]$. An $s_{\partial^{-}}$ triangulation of $(X, \partial X)$ is an $s$-triangulation of the pair $(X, \partial X)$

$$
(f, \partial f):(M, \partial M) \rightarrow(X, \partial X)
$$

for which the restriction $\partial f$ is a homeomorphism. Two $s_{\partial}$-triangulations

$$
\left(f_{i}, \partial f_{i}\right):\left(M_{i}, \partial M_{i}\right) \rightarrow(X, \partial X), i=0,1
$$

are concordant if there exists an $s$-triangulation (7) of the 4-ad and

$$
V=\partial M_{0} \times I, \quad g=\partial f_{0} \times I: V \rightarrow \partial X \times I .
$$

The set of concordance classes is denoted by $\mathcal{S}^{\partial}(X, \partial X)$.

For a compact manifold with boundary $X \hookleftarrow \partial X$ we can write down the following diagram of structures:

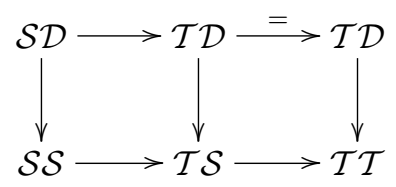

in which, similar to diagram (3), the arrows provide natural maps of "weakening of the structure' (cf. $[\mathbf{4 , 9}]$ and $[\mathbf{1 0}]$ ), and we can interpret every arrow (or their composition) as a relation of a partial order. 
For any structure in (9), the right symbol which corresponds to the structure on the boundary is 'stronger than or equal' to the left symbol. Every arrow in (9) gives a natural map of 'weakening of the structure' on a manifold with boundary $(X, \partial X)$. From this it follows that any sequence of arrows from (9) also gives a map of 'weakening of the structure'. Hence, for any $(X, \partial X)$, diagram (9) provides the following commutative diagram of structure sets (compare with the diagram on page 116 of $[\mathbf{1 0}])$ :

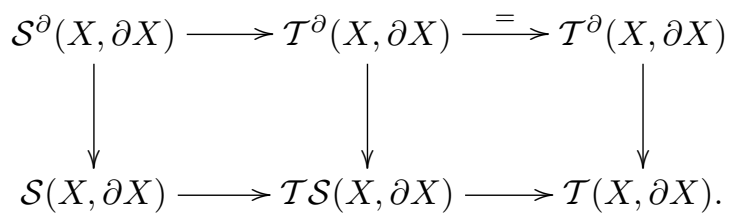

Diagram (10) gives an opportunity to construct several commutative diagrams which have the form of the square or of the triangle; for example we have

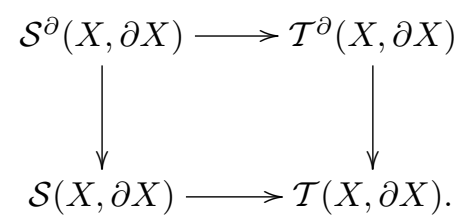

Excluding the trivial cases when the restriction of the structure to the boundary is a homeomorphism, we obtain the following relations between structures on $(X, \partial X)$ and structures on $\partial X$ (cf. $[\mathbf{4 , 5}, \mathbf{9}]$ and $[\mathbf{1 0}])$ :

$$
\mathcal{S S} \rightarrow \mathcal{S}, \quad \mathcal{T} \mathcal{S} \rightarrow \mathcal{S}, \quad \mathcal{T} \mathcal{T} \rightarrow \mathcal{T}
$$

In (12) the left pairs of symbols correspond to the structures on $(X, \partial X)$ and the right symbols correspond to the structures on $\partial X$ which is the restriction of the structure on $(X, \partial X)$ to the boundary. The relations in (12) and diagram (9) provide the following diagram of structures (cf. $[\mathbf{4}, \mathbf{9}]$ and $[\mathbf{1 0}])$ :

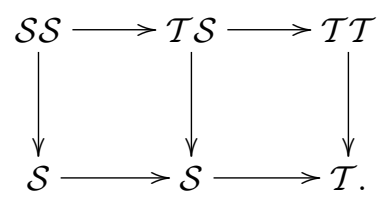

As above, for any manifold with boundary $(X, \partial X)$, diagram (13) provides the following commutative diagram of structure sets (compare with the diagram on page 116 of $[\mathbf{1 0}])$ :

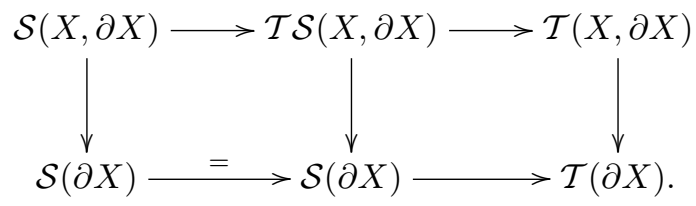




\section{The structure sets of a closed manifold pair $(X, Y)$}

Let $\left(X^{n}, Y^{n-q}\right)$ be a closed manifold pair without boundary. In this section we systematically describe various structures on this pair. From our description, it follows that, for a pair of closed manifolds, the structures and relations between them are similar to the structures and relations for a manifold with boundary. In fact it is clear from our consideration, that in a number of cases the submanifold 'plays the role of a boundary' [2]. For a pair $\left(X^{n} \hookleftarrow Y^{n-q}\right)$, the list of structures almost coincides with the corresponding list of structures for $(X, \partial X)$. Below we explain the difference. First we remark that all structures from (4) are realized for manifold pairs $(X, Y)$. Now we define these structures (cf. $[\mathbf{2}, \mathbf{9}]$ and $[\mathbf{1 0}])$.

$(\mathcal{T} \mathcal{T})$. For a closed manifold pair $(X, Y)$, this structure is given by the structure set $\mathcal{T}(X, Y)=\mathcal{T}(X)$ (cf. [9]) and it consists of concordance classes of $t$-triangulations of the manifold $X$.

$(\mathcal{T S})$. This structure is given by the structure set $\mathcal{N S}(X, Y)$ introduced in [2]. Its restriction to $X$ gives the $\mathcal{T}$ structure on $X$ and its restriction to $Y$ gives the $\mathcal{S}$-structure $[2]$. This structure is similar to the mixed structure on the manifold with boundary (cf. $[\mathbf{2}, \mathbf{4}]$ and $[\mathbf{1 0}]$ ).

Let $f: M \rightarrow X$ be a $t$-triangulation which is transversal to the submanifold $Y$ with $N=f^{-1}(Y)$, and the restriction $\left.f\right|_{N}: N \rightarrow Y$ is a simple homotopy equivalence. Two such maps,

$$
f_{i}: M_{i} \rightarrow X, \quad N_{i}=f_{i}^{-1}(Y),(i=1,2),
$$

are concordant if there exists a $t$-triangulation

$$
\left(F ; f_{0}, f_{1}\right):\left(W ; M_{0}, M_{1}\right) \rightarrow(X \times I ; X \times\{0\}, X \times\{1\})
$$

with the following properties:

(i) $\partial W=M_{1} \cup M_{2}$ and $\left.F\right|_{M_{i}}=f_{i}(i=0,1)$;

(ii) $F$ is transversal to $Y$ with $F^{-1}(Y)=V$ and $\partial V=N_{0} \cup N_{1}$;

(iii) the restriction $\left.F\right|_{V}$ is an $s$-triangulation of the triad

$$
\left(\left.F\right|_{V} ;\left.f_{0}\right|_{N_{0}},\left.f_{1}\right|_{N_{1}}\right):\left(V ; N_{0}, N_{1}\right) \rightarrow(Y \times I ; Y \times\{0\}, Y \times\{1\}) .
$$

The set of equivalence classes of such maps is denoted by $\mathcal{N S}(X, \partial X)$ (cf. [2]).

$(\mathcal{T D})$. The structure set in this case is given by the $\operatorname{rel}_{\partial} t$-triangulations of the manifold with boundary $(X \backslash Y, \partial(X \backslash Y))$, that is $\mathcal{T}^{\partial}(X \backslash Y, \partial(X \backslash Y))($ cf. [4, 5, 9] and $[\mathbf{1 0}])$.

$(\mathcal{S S})$. This structure is given by the structure set $\mathcal{S}(X, Y, \xi)$ where $\xi$ is a topological normal block bundle of $Y$ in $X$ (cf. [9]). The restriction of this structure to $X$ is an $\mathcal{S}$-structure, and the restriction to $Y$ also is an $\mathcal{S}$-structure. We now give an explicit definition following $[\mathbf{9}]$.

An $s$-triangulation of a manifold pair $\mathcal{S}(X, Y, \xi)$ is a $t$-triangulation

$$
f: M \rightarrow X, \quad g=\left.f\right|_{N}: N \rightarrow Y
$$

which is transversal to $Y$ with $N=f^{-1}(Y)$ and for which the maps

$$
g=f_{N}: N \rightarrow Y, \quad \text { and }\left.\quad f\right|_{M \backslash N}=h:(M \backslash N, \partial(M \backslash N)) \rightarrow(X \backslash Y, \partial(X \backslash Y))
$$


are $s$-triangulations (cf. $[\mathbf{2}, \mathbf{5}]$ and $[\mathbf{9}]$ ). Two such $s$-triangulations,

$$
\left(f_{;} g_{i}, h_{i}\right):\left(M_{i} ; N_{i}, M_{i} \backslash N_{i}\right) \rightarrow(X ; Y, X \backslash Y), i=0,1,
$$

are concordant if there exists an $s$-triangulation

$$
(F ; G, H):(W ; V, W \backslash V) \rightarrow(X \times I ; Y \times I,(X \backslash Y) \times I)
$$

with

$$
\begin{gathered}
\partial W=M_{0} \cup M_{1}, \quad \partial V=N_{0} \cup N_{1}, \\
\partial(W \backslash V)=\left(M_{0} \backslash N_{0}\right) \cup\left(M_{1} \backslash N_{1}\right) \cup F^{-1}(\partial(X \backslash Y) \times I), \\
F_{M_{i}}=f_{i}, \quad G_{M_{i}}=g_{i}, \quad H_{M_{i} \backslash N_{i}}=h_{i} .
\end{gathered}
$$

The set of concordance classes is denoted by $\mathcal{S}(X, Y, \xi)$.

$(\mathcal{S D})$. In this case we shall consider $\mathcal{S}$-structures on $X$ whose restriction to a tubular neighborhood of $Y$ provide $\mathcal{D}$-structures. The structure set in this case is given by the $\operatorname{rel}_{\partial} s$-triangulations of the manifold with boundary $(X \backslash Y, \partial(X \backslash Y))$, that is

$$
\mathcal{S}^{\partial}(X \backslash Y, \partial(X \backslash Y))
$$

(cf. $[\mathbf{4}, \mathbf{5}, \mathbf{9}]$ and $[\mathbf{1 0}])$.

The relations (9) take place for the structures introduced above in this section (cf. $[\mathbf{2}, \mathbf{4}, \mathbf{5}, \mathbf{9}]$ and $[\mathbf{1 0}])$. Thus, for a closed manifold pair $(X, Y)$, diagram (9) provides the following commutative diagram of structure sets:

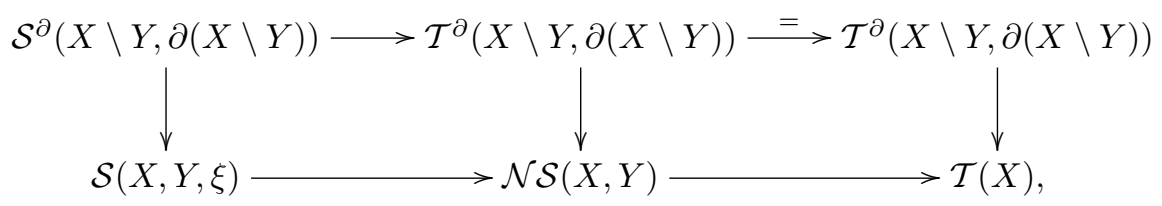

which is similar to diagram (10). In a similar way (cf. $[\mathbf{2}, \mathbf{4}, \mathbf{5}]$ and $[\mathbf{9}]$ ), using the restriction of a structure on the manifold $X$ to the submanifold $Y$, we obtain the relations between structures on $(X, Y)$ and structures on $Y$ given in (12). Now in (12) the left pairs of symbols for any arrow correspond to structures on $(X, Y)$ and the right symbols correspond to the structures on $Y$. The results of $[\mathbf{2}, \mathbf{9}]$, and $[\mathbf{1 0}]$ provide the relations (13) between structures for a closed manifold pair $(X, Y)$. Thus, for any closed manifold pair $(X, Y)$, diagram (13) provides the following commutative diagram of structure sets that is similar to (14):

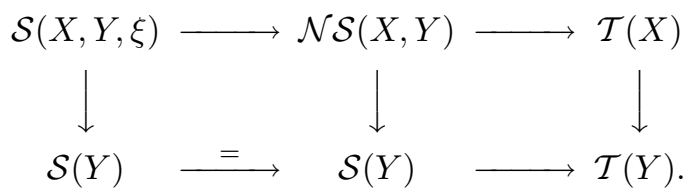

However, there is a difference between structures for the pairs $(X, \partial X)$ and $(X, Y)$. For a manifold pair $(X, Y)$, we can also consider the $\mathcal{S}$-structure on $X$ as $\mathcal{S} \mathcal{T}$-structure on the pair $(X, Y)$. There is no analogue structure for the case of manifolds with boundary. In this case the restriction of the structure to the submanifold gives a 'weaker structure' than the structure on the ambient manifold. This structure fits 
into the following commutative diagram of structures (cf. $[\mathbf{2}]$ and $[\mathbf{9}]$ ):

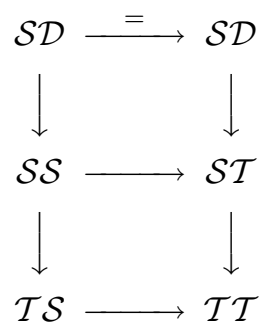

which has no analog for the structures on manifolds with boundary. For any closed manifold pair $(X, Y)$, diagram (17) provides the following commutative diagram of structure sets:

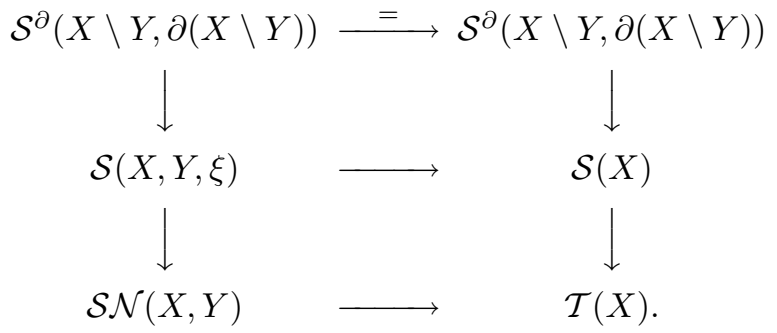

The structure $\mathcal{S} \mathcal{T}$ fits also into the following diagram of structures:

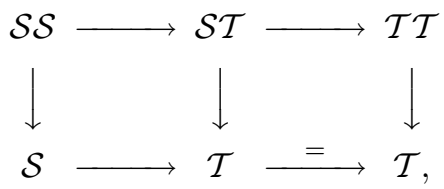

in which the vertical arrows correspond to the restriction of the corresponding structure to the submanifold. For any closed manifold pair $(X, Y)$, diagram (19) gives the following commutative diagram of structure sets:

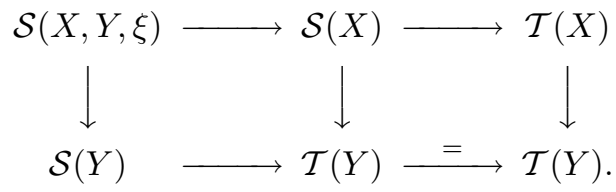

\section{The spectrum level. The diagrams of structure sets}

In accordance with the algebraic surgery theory of Ranicki (cf. $[\mathbf{1}, \mathbf{4}, \mathbf{6}]$ and $[\mathbf{8}]$ ) the structure sets introduced above, various surgery obstruction groups, and natural maps are realized on the spectrum level. We shall use the following notation.

Let $\mathbf{L}_{\bullet}$ be the 1 -connected cover of the simply connected surgery $\Omega$-spectrum $\mathbf{L}_{\bullet}$ (1) with $\mathbf{L}_{\bullet} \simeq G /$ TOP and $\pi_{n}\left(\mathbf{L}_{\bullet}(1)\right)=L_{n}(1)(n>0)$ (cf. [8] and [9]). The cofibration

$$
X_{+} \wedge \mathbf{L} \bullet \rightarrow \mathbb{L}\left(\pi_{1}(X)\right) \rightarrow \mathbb{S}(X)
$$

is defined for any topological space $X$ (cf. $[\mathbf{8}]$ and $[\mathbf{9}]$ ), and the algebraic surgery exact 
sequence

$$
\cdots \rightarrow L_{n+1}\left(\pi_{1}(X)\right) \rightarrow \mathcal{S}_{n+1}(X) \rightarrow H_{n}\left(X ; \mathbf{L}_{\bullet}\right) \rightarrow L_{n}\left(\pi_{1}(X)\right) \rightarrow \cdots
$$

is a homotopy long exact sequence of this cofibration with

$$
\pi_{j}(\mathbb{S}(X))=\mathcal{S}_{j}(X), \quad \mathcal{T}_{j}(X)=H_{j}\left(X ; \mathbf{L}_{\bullet}\right)
$$

The groups $L_{*}\left(\pi_{1}(X)\right)$ are the surgery obstruction groups of Wall [10]. For a closed manifold $X^{n}$ with $n \geqslant 5$, we have

$$
\mathcal{S}(X) \cong \mathcal{S}_{n+1}(X), \quad \mathcal{T}(X) \cong \mathcal{T}_{n}(X)=H_{n}\left(X ; \mathbf{L}_{\bullet}\right)
$$

For the structure sets of a compact manifold $\left(X^{n}, \partial X^{n}\right)$ with boundary $\partial X$ there exists the following homotopy commutative diagram of spectra (cf. $[\mathbf{4}, \mathbf{5}, \mathbf{8}]$ and $[\mathbf{9}]$ )

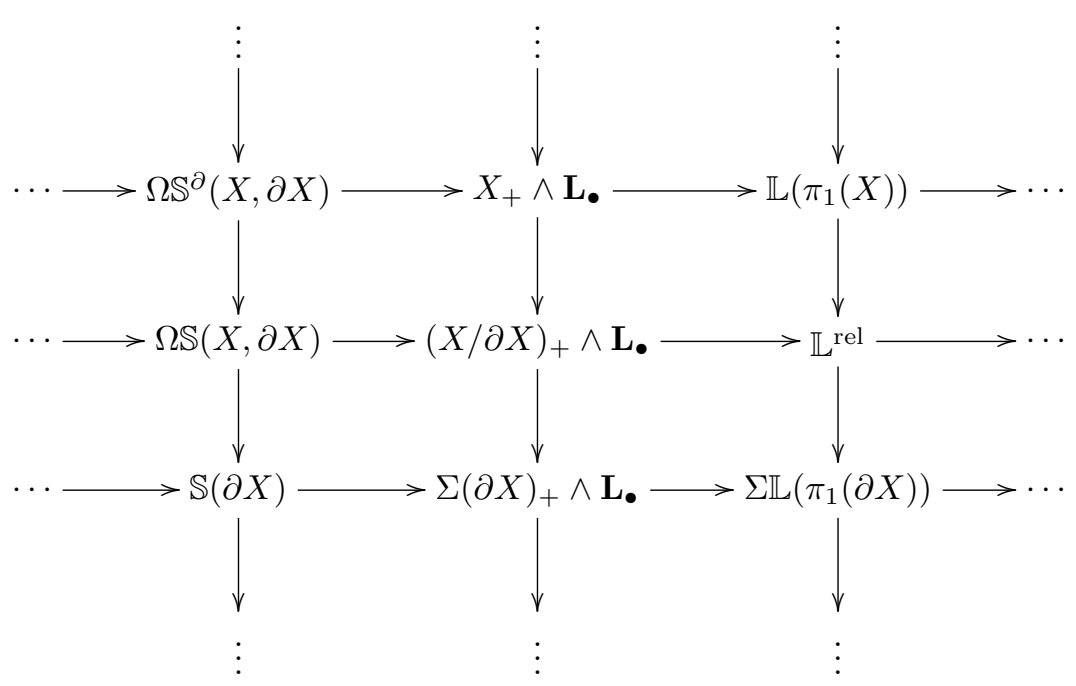

where $\mathbb{L}^{\text {rel }}=\mathbb{L}\left(\pi_{1}(\partial X) \rightarrow \pi_{1}(X)\right)$ is a spectrum for the relative $L$-groups. The homotopy long exact sequences of the maps from (25) give the commutative diagram of exact sequences (cf. [9] and $[\mathbf{1 0}]$ )

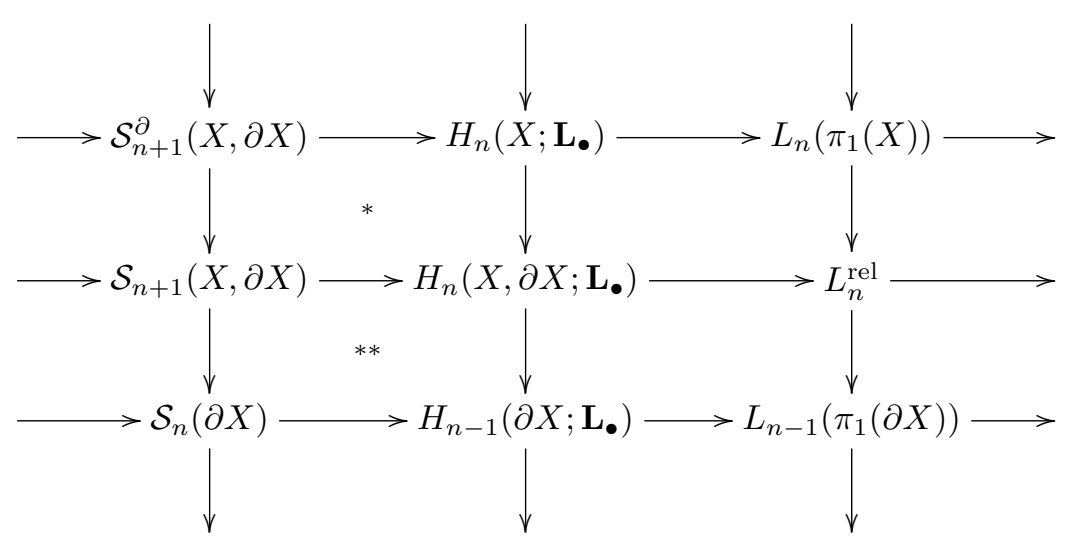


and we have the following isomorphisms of the structure sets:

$$
\begin{aligned}
\mathcal{T}^{\partial}(X, \partial X) & \cong H_{n}\left(X ; \mathbf{L}_{\bullet}\right), & \mathcal{T}(X, \partial X) & \cong H_{*}\left(X, \partial X ; \mathbf{L}_{\bullet}\right), \\
\mathcal{T}(\partial X) & \cong H_{n-1}\left(\partial X ; \mathbf{L}_{\bullet}\right), & \mathcal{S}^{\partial}(X, \partial X) & \cong \mathcal{S}_{n+1}^{\partial}(X, \partial X), \\
\mathcal{S}(X, \partial X) & \cong \mathcal{S}_{n+1}(X, \partial X), & \mathcal{S}(\partial X) & \left.\cong \mathcal{S}_{n}(\partial X)\right) .
\end{aligned}
$$

Note that the commutative square $*$ in $(26)$ is isomorphic to the commutative square

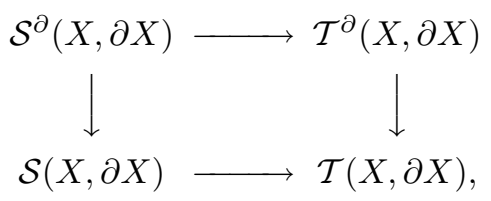

which follows from diagram (10), and the commutative square $* *$ is isomorphic to the commutative square

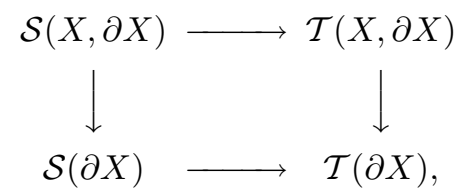

which follows from (14).

For a compact manifold with boundary $\left(X^{n}, \partial X^{n}\right)$, we define a spectrum

$$
\mathbb{T S}(X, \partial X)
$$

as the homotopy cofiber of the map $\mathbb{L}\left(\pi_{1}(X)\right) \longrightarrow \mathbb{S}(X, \partial X)$, which is a composition of maps from diagram (25) [4]. Thus, as follows from diagram (25), the spectrum $\Omega \mathbb{T S}(X, \partial X)$ fits into the following cofibrations (cf. [4]):

$$
\begin{aligned}
& \Omega \mathbb{T S}(X, \partial X) \longrightarrow \mathbb{L}\left(\pi_{1}(X)\right) \longrightarrow \mathbb{S}(X, \partial X), \\
& \Omega \mathbb{T S}(X, \partial X) \longrightarrow(X / \partial X)_{+} \wedge \mathbf{L}_{\bullet} \longrightarrow \Sigma \mathbb{L}\left(\pi_{1}(\partial X)\right), \\
& \Omega \mathbb{T S}(X, \partial X) \longrightarrow \Sigma(\partial X) \quad \longrightarrow \quad\left(X_{+} \wedge \mathbf{L}_{\bullet}\right) .
\end{aligned}
$$

Denote $\pi_{i}(\mathbb{T S}(X, \partial X))=\mathcal{T} \mathcal{S}_{i}(X, \partial X)$, and we have an isomorphism

$$
\mathcal{T} \mathcal{S}(X, \partial X) \cong \mathcal{T} \mathcal{S}_{n+1}(X, \partial X)
$$

The mixed structure sets relate to other structure sets introduced above by the following braids of exact sequences [4]

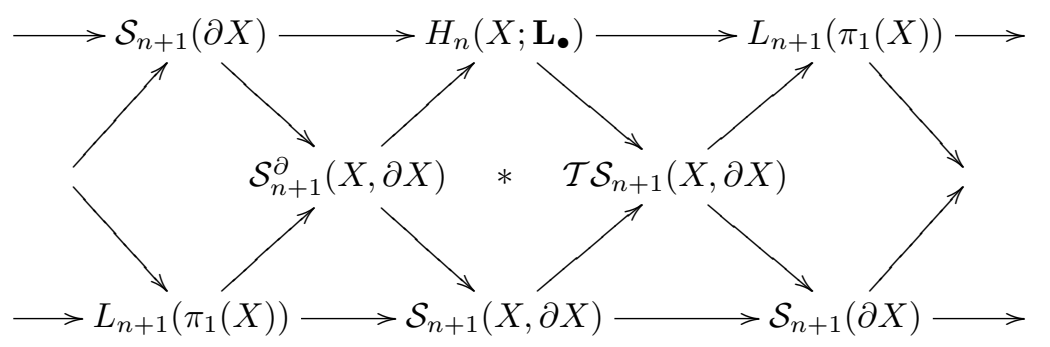




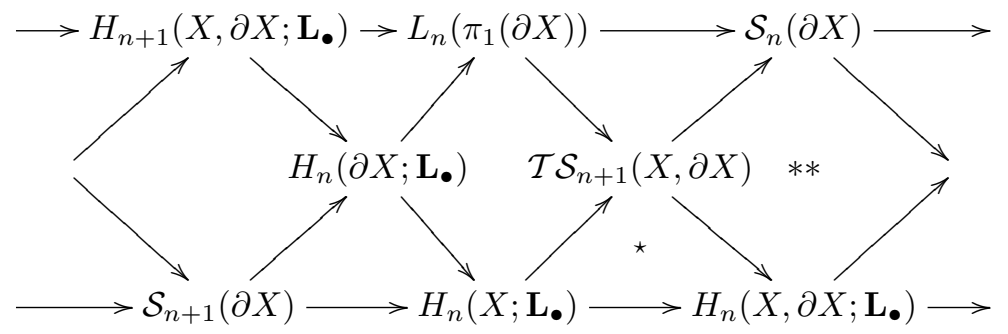

and

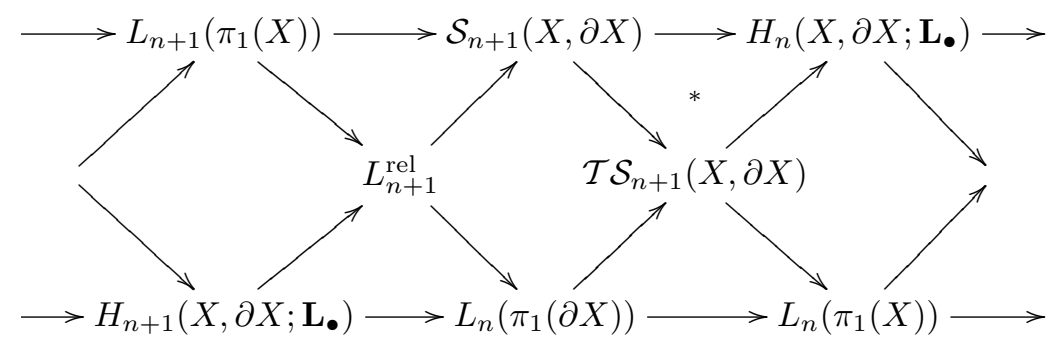

which are realized on the spectrum level.

Note that the commutative square $*$ in (31) is isomorphic to the left commutative square in diagram (10), the commutative square $* *$ in (32) is isomorphic to the right commutative square in diagram (14), the commutative triangle $\star$ in (32) follows from the right square of (10), and the commutative triangle $*$ in diagram (33) is isomorphic to the commutative triangle

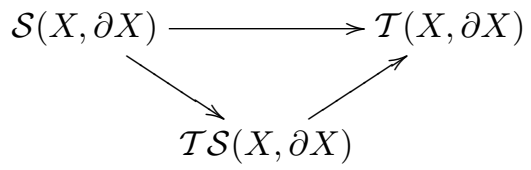

which follows from diagram (14).

Now let $\left(X^{n}, Y^{n-q}, \xi\right)$ be a closed manifold pair (cf. [9, $\left.\left.\S 7.2\right]\right)$. An $s$-triangulation $f: M \rightarrow X$ splits along $Y$ if it is homotopic to a map which is an $s$-triangulation of the manifold pair $(X, Y, \xi)$ (the definition was given in Section 3). The splitting obstruction group $L S_{n-q}(F)$ is defined in $[\mathbf{9}, \S 7.2]$. Let $\partial U$ be the boundary of a tubular neighborhood $U$ of $Y$ in $X$. The groups $L S_{n-q}(F)$ depend only on $n-q \bmod 4$ and a pushout square

$$
F=\left(\begin{array}{ccc}
\pi_{1}(\partial U) & \longrightarrow & \pi_{1}(X \backslash Y) \\
\downarrow & & \downarrow \\
\pi_{1}(Y) & \longrightarrow & \pi_{1}(X)
\end{array}\right)
$$

of fundamental groups with orientations.

If $f: M \rightarrow X$ is a $t$-triangulation then by $[\mathbf{9}, \S 7.2]$ a group $L P_{n-q}(F)$ of obstructions is defined. This group provides obstructions to the existence of an $s$-triangulation of $(X, Y, \xi)$ in the class of the normal bordism of the map $f$. The groups $L P_{n-q}(F)$ depend only on $n-q \bmod 4$ and the square $F$ as well. There exists a homotopy 
commutative diagram of spectra (cf. [2] and [9])

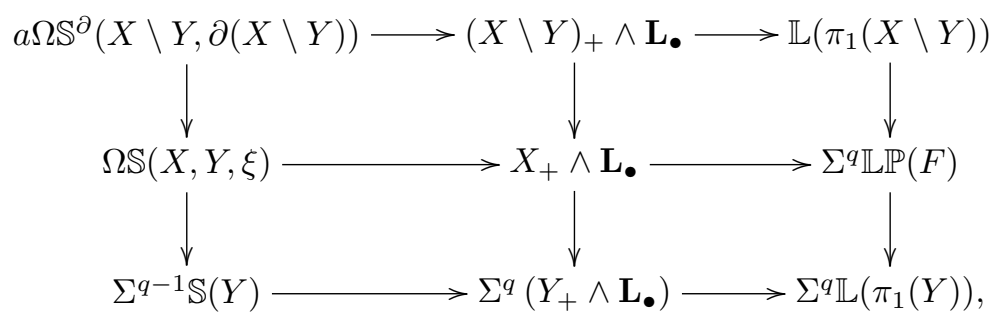

where the spectrum $\mathbb{L P}(F)$ is the spectrum for obstruction groups $L P_{i}(F)$, and all rows and columns in (36) are cofibrations. Denote $\pi_{i}(\mathbb{S}(X, Y, \xi))=\mathcal{S}_{i}(X, Y, \xi)$, and we have an isomorphism [9]

$$
\mathcal{S}_{n+1}(X, Y, \xi) \cong \mathcal{S}(X, Y, \xi)
$$

The homotopy long exact sequences of rows and columns of diagram (36) give the homotopy commutative diagram of groups

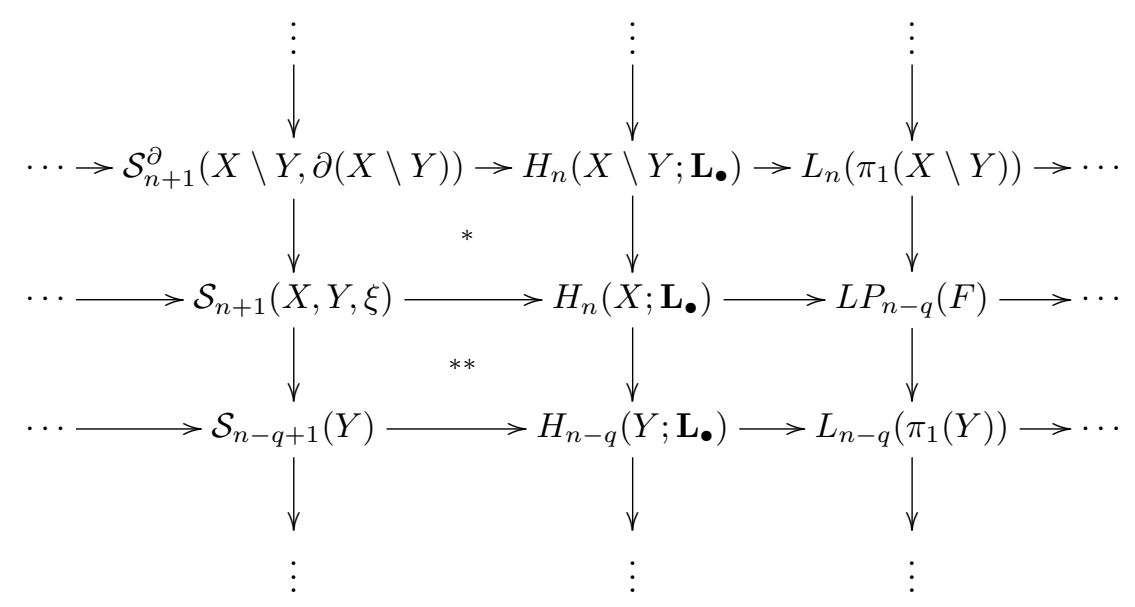

whose rows and columns are exact sequences. This is the diagram of [9, Prop. 7.2.6]. Note that the commutative square $*$ in (38) is isomorphic to the commutative square

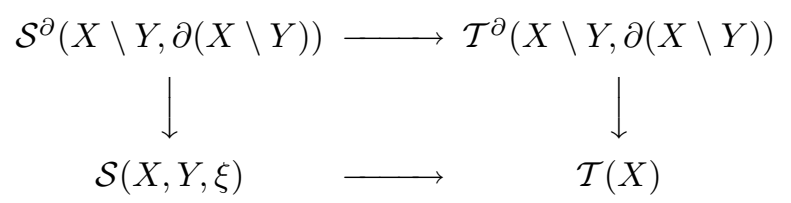

which follows from diagram (15). The commutative square $* *$ in (38) is isomorphic to the commutative square

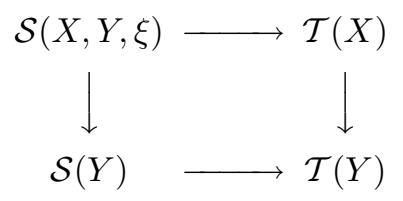


which follows from diagram (19). Thus diagram (38) is similar to diagram (26) which was constructed for a manifold with boundary.

For a closed manifold pair $\left(X^{n}, Y^{n-q}\right)$, we define a spectrum $\mathbb{N} \mathbb{S}(X, Y)$ as the homotopy fiber of the map $\mathbb{L}\left(\pi_{1}(X \backslash Y)\right) \longrightarrow \mathbb{S}(X, Y, \xi)$ which is the composition of maps from the extended diagram (36) [2]. It follows from (36) (cf. [2]) that this spectrum fits into the following cofibrations:

$$
\begin{aligned}
& \mathbb{N} \mathbb{S}(X, Y) \longrightarrow \mathbb{L}\left(\pi_{1}(X \backslash Y)\right) \longrightarrow \mathbb{S}(X, Y, \xi), \\
& \mathbb{N S}(X, Y) \longrightarrow X_{+} \wedge \mathbf{L}_{\bullet} \longrightarrow \quad \Sigma^{q} \mathbb{L}\left(\pi_{1}(Y)\right), \\
& \mathbb{N} \mathbb{S}(X, Y) \longrightarrow \Sigma^{q-1} \mathbb{S}(Y) \longrightarrow \Sigma\left((X \backslash Y)_{+} \wedge \mathbf{L}_{\bullet}\right) .
\end{aligned}
$$

Note that the cofibrations in $(41)$ for $(X, Y)$ are similar to cofibrations in (30) for $(X, \partial X)$. Denote $\pi_{i}(\mathbb{N} S(X, Y))=\mathcal{N} \mathcal{S}_{i}(X, Y)$, and we have an isomorphism

$$
\mathcal{N S}(X, Y) \cong \mathcal{N S}_{n}(X, Y) \text {. }
$$

The structure sets $\mathcal{N S}(X, Y)$ relate to the other structure sets for a manifold pair $(X, Y)$ by the following braids of exact sequences $[\mathbf{2}]$

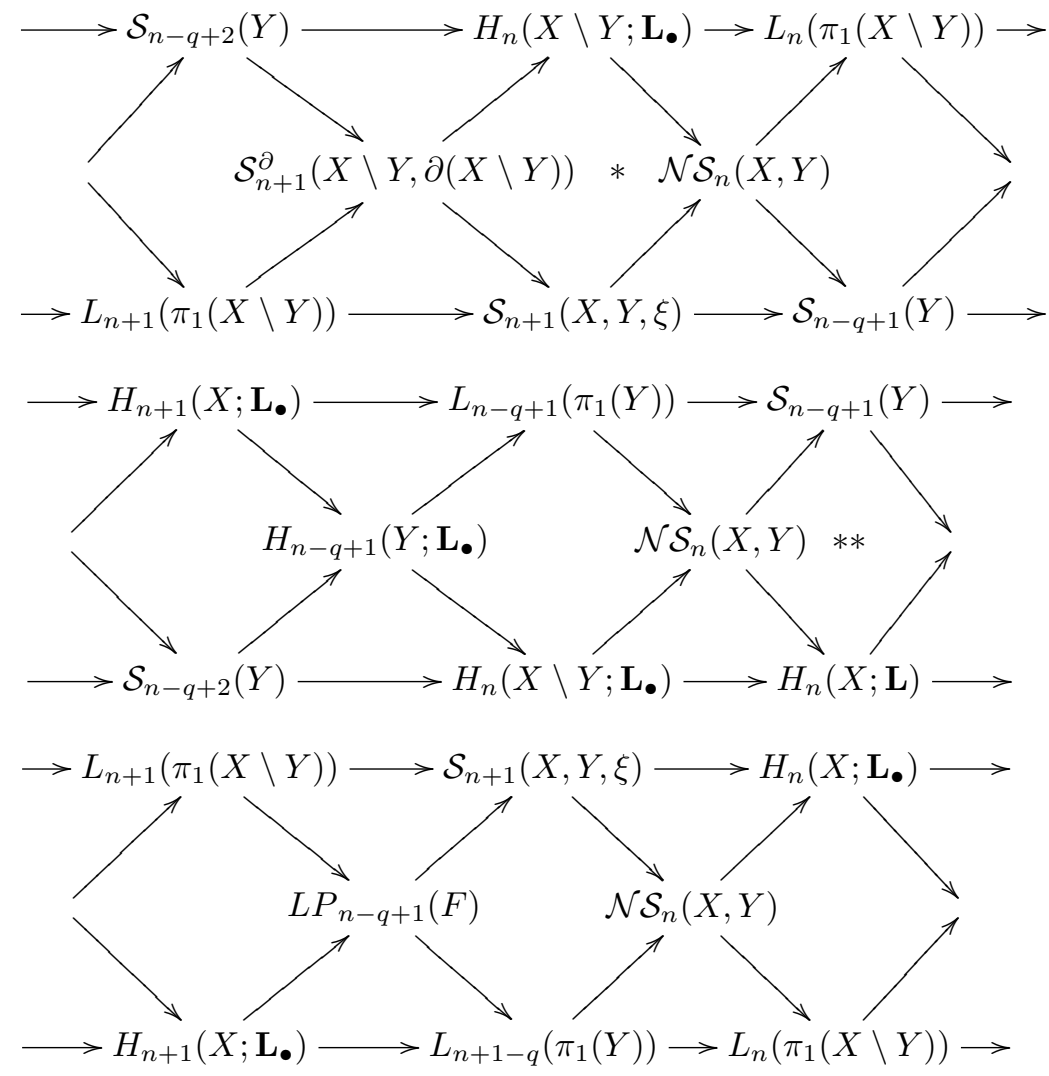

Diagrams (42)-(44) are realized on the spectrum level and follow from diagrams (36) (cf. $[\mathbf{1}, \mathbf{2}]$ and [7]). Diagram (42) is similar to diagram(31), diagram (43) is similar to diagram (32), and diagram (44) is similar to diagram (33). 
For the case of a manifold pair $(X, Y, \xi)$ we can consider the following pair of topological spaces $(X, X \backslash Y)$, where as usual $X \backslash Y$ denotes the closure of $X \backslash U$ where $U$ is a tubular neighborhood of $Y$ in $X$. Then the commutative diagram of exact sequences from page 560 of [9] gives the diagram

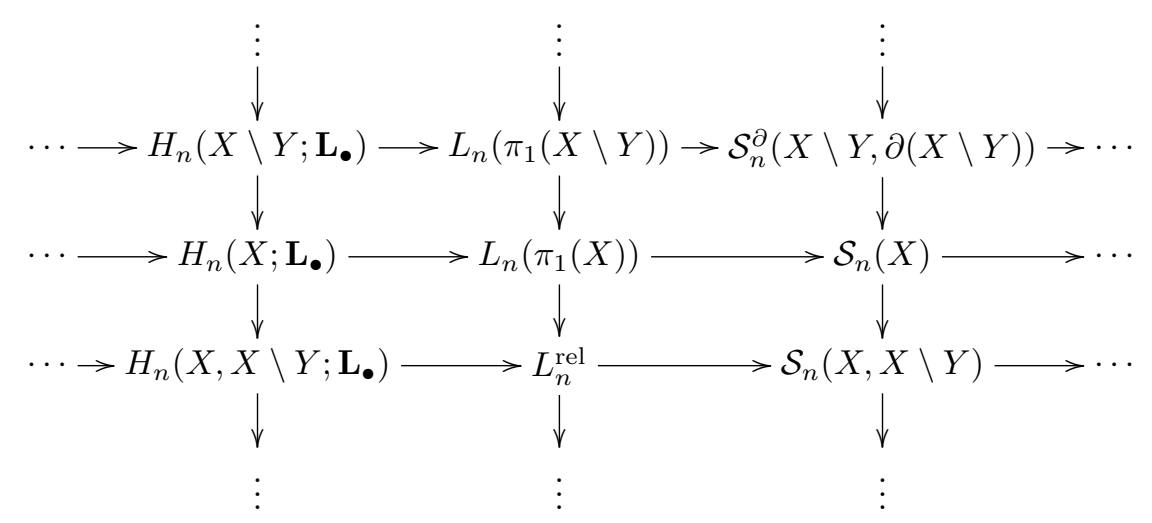

where

$$
L_{n}^{\mathrm{rel}}=L_{n}\left(\pi_{1}(X \backslash Y) \rightarrow \pi_{1}(X)\right), \quad H_{n}\left(X, X \backslash Y ; \mathbf{L}_{\bullet}\right) \cong H_{n-q}\left(Y ; \mathbf{L}_{\bullet}\right) .
$$

The structure sets for a manifold pair $(X, Y)$ also fit into the following commutative diagrams of exact sequences $[\mathbf{9}, \S 7.2]$ :
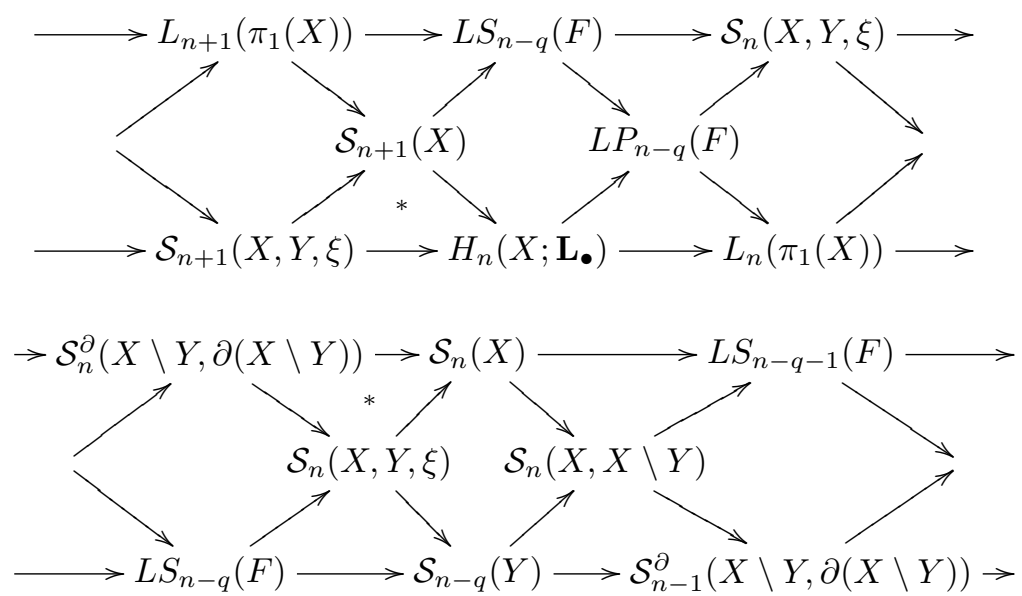

and

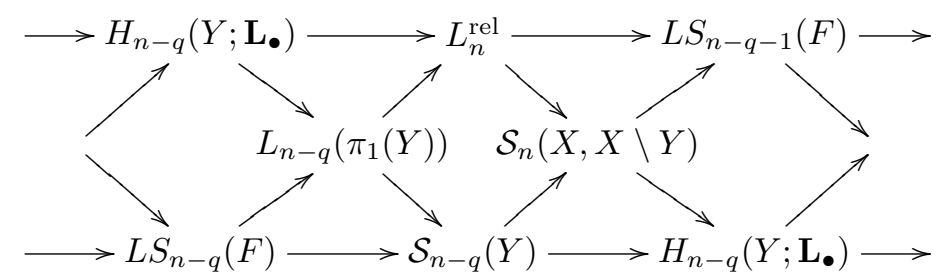


which are realized on the spectrum level. The commutative triangle $*$ in (46) corresponds to the following diagram of structures

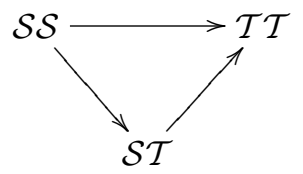

which follows from diagram (17).

The commutative triangle $*$ in (47) corresponds to the following diagram of structures

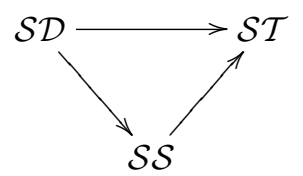

which follows from diagram (17).

For the case $(X, \partial X)$, there are no diagrams that are similar to diagrams (45)-(48). In this case we have a simple homotopy equivalence $(X \backslash \partial X) \rightarrow X$, and the theory is degenerated.

\section{The structure sets of a manifold pair with boundary}

In this section we describe various structure sets which arise naturally for a compact manifold pair with boundary in (1). We shall use notation that is similar to the notation of previous sections. Thus, for example,

$$
\left[\begin{array}{ll}
\mathcal{T} & \mathcal{T} \\
\mathcal{S} & \mathcal{D}
\end{array}\right]
$$

denotes a structure on $Q$ in $(1)$ which is a $\mathcal{T} \mathcal{T}$-structure on $(X, \partial X)$ whose restriction to $(Y \hookleftarrow \partial Y)$ (the bottom pair of symbols) gives an $\mathcal{S D}$-structure, and whose restriction to $(\partial X \hookleftarrow \partial Y)$ (right vertical pair of symbols) gives a $\mathcal{T} \mathcal{D}$-structure.

We shall denote by $F$ the pushout square for the manifold pair $(X, Y)$ as in (35). The manifold pair with boundary $Q$ in (1) defines a pair of closed manifolds $\partial Y \subset \partial X$ with a pushout square

$$
F_{\partial}=\left(\begin{array}{ccc}
\pi_{1}(S(\partial \xi)) & \longrightarrow & \pi_{1}(\partial X \backslash \partial Y) \\
\downarrow & & \downarrow \\
\pi_{1}(\partial Y) & \longrightarrow & \pi_{1}(\partial X)
\end{array}\right)
$$

of fundamental groups for the splitting problem. A natural inclusion $\delta: \partial X \rightarrow X$ induces a map $\Delta: F_{\partial} \rightarrow F$ of squares of fundamental groups. The relative splitting obstruction groups $L S_{*}(\Delta)$ and the relative surgery obstruction groups $L P_{*}(\Delta)$ are defined in $[\mathbf{3}, \mathbf{5}, \mathbf{9}]$, and $[\mathbf{1 0}]$.

First we describe structures for which there is a similarity between the pairs $(X, \partial X)$ and $(X, Y)$. In this case for a structure $\left[\begin{array}{l}\mathcal{A B} \\ \mathcal{C} \mathcal{E}\end{array}\right]$ we have that $\mathcal{B}$ and $\mathcal{C}$ are 
stronger than or equal to $\mathcal{A}$, and $\mathcal{E}$ is stronger than or equal to $\mathcal{B}$ and $\mathcal{C}$. In such a way it is sufficiently easy to describe all possible natural structures on a manifold pair with boundary in (1) (cf. [5] and [9, pp. 585-587]). In this case we obtain the following list of possible structures:

$$
\begin{aligned}
& {\left[\begin{array}{l}
\mathcal{S D} \\
\mathcal{D D}
\end{array}\right], \quad\left[\begin{array}{l}
\mathcal{S S} \\
\mathcal{D D}
\end{array}\right], \quad\left[\begin{array}{l}
\mathcal{S D} \\
\mathcal{S D}
\end{array}\right], \quad\left[\begin{array}{l}
\mathcal{S} \mathcal{S} \\
\mathcal{S D}
\end{array}\right], \quad\left[\begin{array}{l}
\mathcal{S S} \\
\mathcal{S S}
\end{array}\right],} \\
& {\left[\begin{array}{l}
\mathcal{T} \mathcal{D} \\
\mathcal{D D}
\end{array}\right], \quad\left[\begin{array}{l}
\mathcal{T} \mathcal{T} \\
\mathcal{D D}
\end{array}\right], \quad\left[\begin{array}{l}
\mathcal{T} \mathcal{D} \\
\mathcal{T} \mathcal{D}
\end{array}\right], \quad\left[\begin{array}{l}
\mathcal{T} \mathcal{T} \\
\mathcal{T} \mathcal{D}
\end{array}\right],\left[\begin{array}{l}
\mathcal{T} \mathcal{T} \\
\mathcal{T} \mathcal{T}
\end{array}\right],} \\
& {\left[\begin{array}{c}
\mathcal{T S} \\
\mathcal{S S}
\end{array}\right], \quad\left[\begin{array}{l}
\mathcal{T} \mathcal{T} \\
\mathcal{S S}
\end{array}\right], \quad\left[\begin{array}{c}
\mathcal{T} \mathcal{S} \\
\mathcal{T S}
\end{array}\right], \quad\left[\begin{array}{l}
\mathcal{T} \mathcal{T} \\
\mathcal{T} \mathcal{S}
\end{array}\right],} \\
& {\left[\begin{array}{c}
\mathcal{T} \mathcal{S} \\
\mathcal{D D}
\end{array}\right], \quad\left[\begin{array}{l}
\mathcal{T} \mathcal{D} \\
\mathcal{S D}
\end{array}\right], \quad\left[\begin{array}{l}
\mathcal{T} \mathcal{S} \\
\mathcal{S D}
\end{array}\right], \quad\left[\begin{array}{l}
\mathcal{T} \mathcal{T} \\
\mathcal{S D}
\end{array}\right], \quad\left[\begin{array}{c}
\mathcal{T} \mathcal{S} \\
\mathcal{T} \mathcal{D}
\end{array}\right] .}
\end{aligned}
$$

Note that the structures in the lists (52)-(55) are partially ordered, which is similar to (3) and (9). A structure

$$
P_{1}=\left[\begin{array}{l}
\mathcal{A}_{1} \mathcal{B}_{1} \\
\mathcal{C}_{1} \mathcal{E}_{1}
\end{array}\right]
$$

is 'stronger' than a structure

$$
P_{2}=\left[\begin{array}{l}
\mathcal{A}_{2} \mathcal{B}_{2} \\
\mathcal{C}_{2} \mathcal{E}_{2}
\end{array}\right]
$$

if $P_{1} \neq P_{2}$ and any element fitting into $P_{1}$ is 'stronger than or equal' to the corresponding element from $P_{2}$ in the sense of (3) and (9). In this case the natural map $P_{1} \rightarrow P_{2}$ of 'weakening of the structure' is defined. In particular, this means that, for any manifold $Q$ in (1), we have a map of structure sets $P_{1}(Q) \rightarrow P_{2}(Q)$.

We can also give the list of possible structures, which correspond to the 'exceptional' structure $\mathcal{S} \mathcal{T}$ on $(X, Y)$ or on $(\partial X, \partial Y)$, similar to the end of Section 3:

$$
\left[\begin{array}{c}
\mathcal{S D} \\
\mathcal{T} \mathcal{D}
\end{array}\right], \quad\left[\begin{array}{c}
\mathcal{S S} \\
\mathcal{T} \mathcal{D}
\end{array}\right], \quad\left[\begin{array}{c}
\mathcal{S S} \\
\mathcal{T S}
\end{array}\right], \quad\left[\begin{array}{c}
\mathcal{S S} \\
\mathcal{T} \mathcal{T}
\end{array}\right], \quad\left[\begin{array}{c}
\mathcal{T S} \\
\mathcal{T} \mathcal{T}
\end{array}\right]
$$

First we remark that several structures from (52)-(56) coincide with structures which were introduced above for the case $(X, \partial X)$. It is easy to see that these are the following structures:

$$
\left[\begin{array}{l}
\mathcal{S D} \\
\mathcal{D D}
\end{array}\right], \quad\left[\begin{array}{c}
\mathcal{T} \mathcal{D} \\
\mathcal{D D}
\end{array}\right], \quad\left[\begin{array}{c}
\mathcal{T} \mathcal{D} \\
\mathcal{T} \mathcal{D}
\end{array}\right], \quad\left[\begin{array}{l}
\mathcal{S D} \\
\mathcal{T} \mathcal{D}
\end{array}\right], \quad\left[\begin{array}{c}
\mathcal{T} \mathcal{T} \\
\mathcal{T} \mathcal{T}
\end{array}\right], \quad\left[\begin{array}{c}
\mathcal{S S} \\
\mathcal{T} \mathcal{T}
\end{array}\right], \quad\left[\begin{array}{c}
\mathcal{T} \mathcal{S} \\
\mathcal{T} \mathcal{T}
\end{array}\right]
$$

Note that it is sufficiently easy to give a direct definition of other structure sets from (52)-(56), similar to Sections 2 and 3. The naturality of the introduced structures (cf. $[\mathbf{2}, \mathbf{4}, \mathbf{5}, \mathbf{8}, \mathbf{9}]$ and $[\mathbf{1 0}]$ ) gives us an opportunity to construct several commutative diagrams of structures which provide the diagrams of structure sets, which are realized on the spectrum level, similar to diagrams (9), (13), (17), and (19).

Next we give several examples of such definitions and constructions of commutative diagrams. 
Example 5.1. Here we consider structures which arise naturally in the rel-case for (1) (cf. $[\mathbf{4}, \mathbf{5}, \mathbf{8}, \mathbf{9}]$ and $[\mathbf{1 0}])$. These are the structures on $Q$ in (1) which have the form

$$
\left[\begin{array}{l}
* \mathcal{D} \\
* \mathcal{D}
\end{array}\right] \text {. }
$$

In particular, the first four structures in (57) are the structures of this type. The first two coincide with the structures $\mathcal{S D}$ and $\mathcal{T} \mathcal{D}$, respectively, on the manifold with boundary $(X \backslash Y, \partial(X \backslash Y))$. The third structure is a $\mathcal{T} \mathcal{D}$-structure on $(X, \partial X)$. The fourth structure is an $\mathcal{S D}$-structure on $(X, \partial X)$.

The $\left[\begin{array}{l}\mathcal{S D} \\ \mathcal{S D}\end{array}\right]$-structure on a pair in (1) corresponds to rel ${ }_{\partial}$-case structure set

$$
\mathcal{S}^{\partial}(X, Y, \xi)=\mathcal{S}^{\partial}(X, Y, \xi ; \partial X)
$$

defined in [9] (cf. also [5]). In this case, consider an $s$-triangulation

$$
(f, \partial f):(M, \partial M) \rightarrow(X, \partial X)
$$

which is transversal to $(Y, \partial Y)$ with a transversal premiere $(N, \partial N)$ such that

$$
\partial f: \partial M \rightarrow \partial X
$$

is a homeomorphism and the maps

$$
g=f_{N}: N \rightarrow Y, \quad \text { and }\left.\quad f\right|_{M \backslash N}=h:(M \backslash N, \partial(M \backslash N)) \rightarrow(X \backslash Y, \partial(X \backslash Y))
$$

are $s$-triangulations (cf. $[\mathbf{2}, \mathbf{5}]$ and $[\mathbf{9}]$ ). The set of concordance classes rel boundary of maps in (59) gives the structure set in (58).

Using the same line of argument as in Section 4 (cf. also [2] and [5]), we can define $\left[\begin{array}{l}\mathcal{T} \mathcal{D} \\ \mathcal{S D}\end{array}\right]$-structures on a pair in (1), which corresponds to the structure set $\mathcal{N} \mathcal{S}^{\partial}(X, Y)$ $(\mathcal{N S}$-structure set relative boundary, as usual we suppose that the restriction to the boundary is a homeomorphism).

Definition 5.2. For a manifold pair $Q$ in (1), let

$$
(f, \partial f):(M, \partial M) \rightarrow(X, \partial X)
$$

be a $t$-triangulation with a homeomorphism $\partial f$. Let $f$ be transversal to $(Y, \partial Y)$ with $(N, \partial N)=f^{-1}(Y, \partial Y)$, and the restriction $g=\left.f\right|_{N}: N \rightarrow Y$ is a simple homotopy equivalence. Two such maps

$$
f_{i}: M_{i} \rightarrow X, \quad N_{i}=f_{i}^{-1}(Y), \quad g_{i}=\left.f_{i}\right|_{N_{i}}(i=0,1),
$$

are concordant if there exists a $t$-triangulation

$$
\left(F ; G, f_{0}, f_{1}\right):\left(W ; M_{0}, M_{1}\right) \rightarrow(X \times I ; \partial X \times I, X \times\{0\}, X \times\{1\})
$$

with the following properties:

(i) $\partial W=\partial M_{0} \times I \cup M_{0} \cup M_{1}$ and $\left.F\right|_{M_{i}}=f_{i}(i=0,1)$;

(ii) $G=g_{0} \times \mathrm{I} d: \partial M_{0} \times I \rightarrow \partial X \times I$;

(iii) $F$ is transversal to $Y \times I$ with $F^{-1}(Y)=V$ and $\partial V=\partial N_{0} \times I \cup N_{0} \cup N_{1}$; 
(iv) the restriction $\left.F\right|_{V}$ is an $s$-triangulation of the 4 -ad

$$
\left(\left.F\right|_{V} ;\left.G\right|_{\partial N_{0} \times I},\left.f_{0}\right|_{N_{0}},\left.f_{1}\right|_{N_{1}}\right):\left(V ; N_{0}, N_{1}\right) \rightarrow(Y \times I ; \partial Y \times I, Y \times\{0\}, Y \times\{1\})
$$

with

$$
\left.G\right|_{\partial N_{0} \times I}=\left.g_{0}\right|_{\partial N_{0}} \times \mathrm{I} d: \partial N_{0} \times I \rightarrow \partial Y \times I .
$$

The set of equivalence classes of such maps is denoted by $\mathcal{N S}^{\partial}(X, Y)$ (cf. [2]).

Similar to Section 4, we can describe the relations between the structure sets of Example 5.1. The structure set $\mathcal{S}^{\partial}(X, Y, \xi)$ is realized on the spectrum level (cf. [5] and $[\mathbf{9}])$ by a spectrum $\mathbb{S}^{\partial}(X, Y, \xi)$ with

$$
\pi_{i}\left(\mathbb{S}^{\partial}(X, Y, \xi)\right)=\mathcal{S}_{i}^{\partial}(X, Y, \xi) \text { and } \mathcal{S}_{n+1}^{\partial}(X, Y, \xi)=\mathcal{S}^{\partial}(X, Y, \xi) .
$$

This spectrum fits into the commutative diagram of cofibrations (cf. [5] and [9])

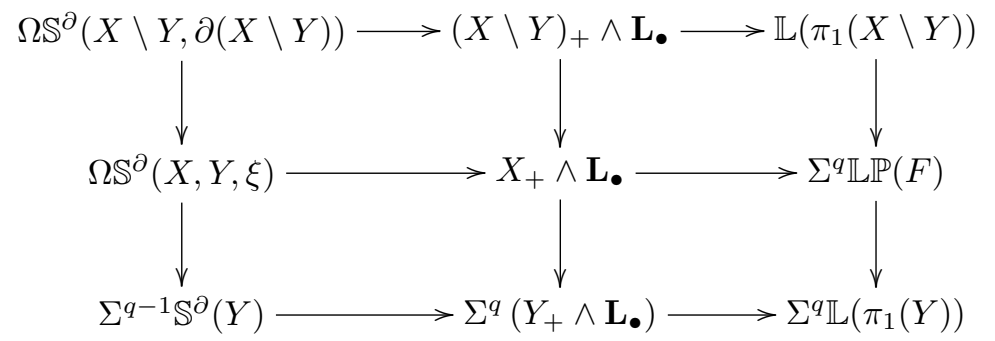

which is rel ${ }_{2}$-version of diagram (36). The homotopy long exact sequences of (60) provides the commutative diagram of exact sequences

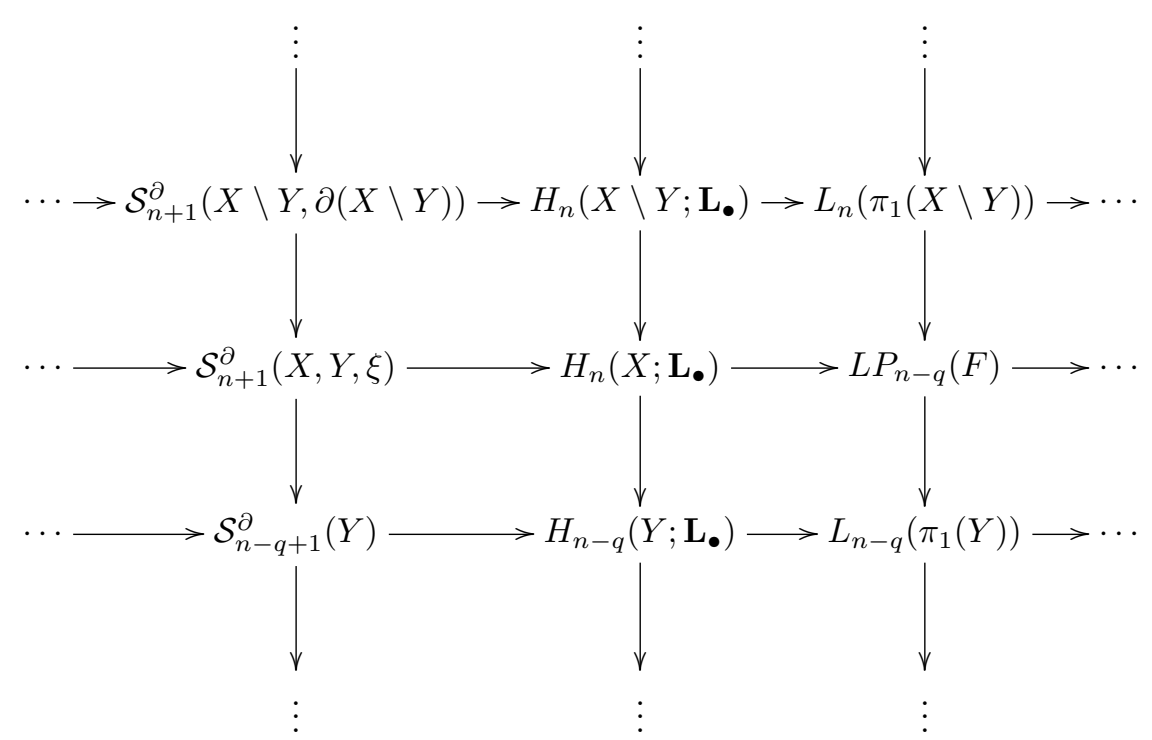

which is the rel ${ }_{\partial}$-version of diagram (38). 
Two left squares in (61) correspond to the following commutative diagram of structures:

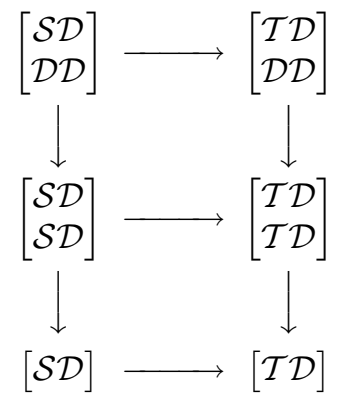

in which all arrows of the upper square and the bottom arrow correspond to the 'weakening of structures' and the bottom vertical arrows correspond to the restrictions of the structures to the submanifold $(Y, \partial Y)$.

Theorem 5.3. There exists a spectrum $\mathbb{N S}^{\partial}(X, Y)$ with homotopy groups

$$
\begin{aligned}
\pi_{i}\left(\mathbb{N S}^{\partial}(X, Y)\right) & =\mathcal{N} \mathcal{S}_{i}^{\partial}(X, Y), \\
\mathcal{N} \mathcal{S}^{\partial}(X, Y) & =\mathcal{N} \mathcal{S}_{n}^{\partial}(X, Y) .
\end{aligned}
$$

Proof. We can use diagram (60), similarly to diagram (36), to define a spectrum $\mathbb{N S}^{\partial}(X, Y)$ fitting into the cofibrations

$$
\begin{aligned}
& \mathbb{N S}^{\partial}(X, Y) \longrightarrow \mathbb{L}\left(\pi_{1}(X \backslash Y)\right) \longrightarrow \mathbb{S}^{\partial}(X, Y, \xi), \\
& \mathbb{N} \mathbb{S}^{\partial}(X, Y) \longrightarrow \Sigma^{q} \mathbb{L}\left(\pi_{1}(Y)\right), \\
& \left.\mathbb{N} \mathbb{S}^{\partial}(X, Y) \longrightarrow \mathbf{L}_{\bullet} \longrightarrow \Sigma^{q-1} \mathbb{S}^{\partial}(Y) \longrightarrow(X \backslash Y)_{+} \wedge \mathbf{L}_{\bullet}\right),
\end{aligned}
$$

which are similar to the cofibrations in (41). From here the result follows using the standard argument (cf. $[\mathbf{2}, \mathbf{5}, \mathbf{9}]$ and $[\mathbf{1 0}]$ ).

Theorem 5.4. The groups $\mathcal{N S}_{i}^{\partial}(X, Y)$ fit into the following braids of exact sequences:
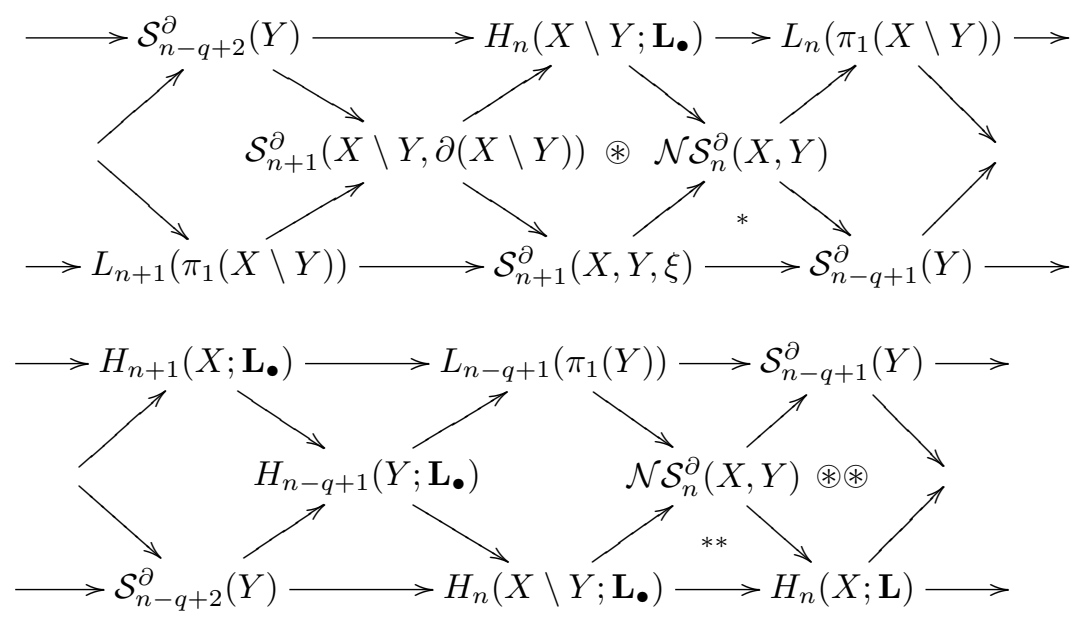


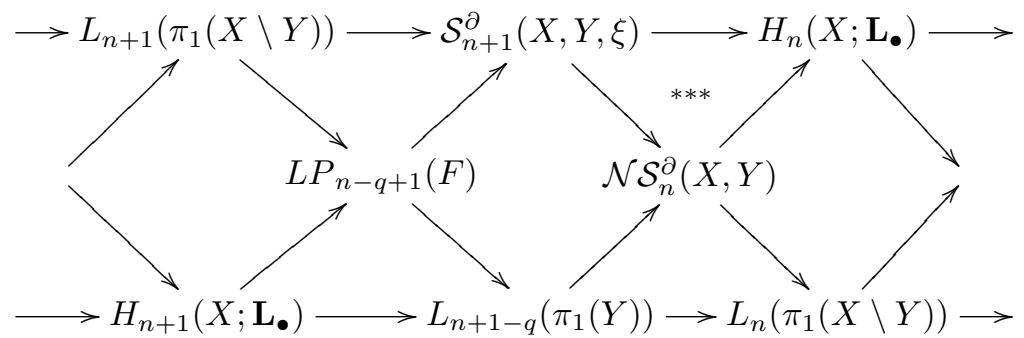

which are realized on the spectrum level.

Proof. The result follows from diagram (60), similar to the construction of diagram (42)-(44) (cf. [1, 2] and [7]).

Note that diagrams (64)-(66), in particular, give several natural relations between structures from (52)-(57) for (1) and structures for (Y, $\partial Y)$ (cf., e.g., the commutative triangles $*, * *, * * *$, and the commutative squares $\circledast$ and $\circledast \circledast)$.

Example 5.5. To understand some of the structures from (52)-(56) we need to use structures on manifold triads [10]. Let $\mathcal{X}=\left(X ; \partial_{0} X, \partial_{1} X\right)$ be a manifold triad [10], where

$$
\partial X=\partial_{0} X \cup \partial_{1} X \text { and let } \partial_{\emptyset} X=\partial_{1} X \cap \partial_{0} X .
$$

We can write down this triad in the following form:

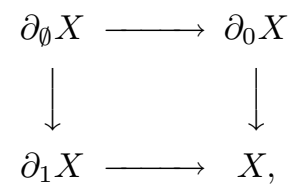

where all the maps are induced by inclusions. In this case, the results from Section 10 of $[\mathbf{1 0}]$ (cf. also [8] and [9]) provide the following structure sets:

- $\mathcal{T}(\mathcal{X})$ - the set of classes of normal bordisms of $t$-triangulations of $\mathcal{X}$,

- $\mathcal{S}(\mathcal{X})$ - the set of classes of $s$-triangulations of $\mathcal{X}$,

- $\mathcal{T}^{\partial_{1} X}(\mathcal{X})$ - the set of classes of normal bordisms of $t$-triangulations of $\mathcal{X}$ rel $\partial_{1} X$,

- $\mathcal{S}^{\partial_{1} X}(\mathcal{X})$ - the set of classes of $s$-triangulations of $\mathcal{X} \operatorname{rel} \partial_{1} X$,

and various structure sets of the pair $\left(\partial_{1} X, \partial_{\emptyset} X\right)$.

Let

$$
F=\left(\begin{array}{ccc}
\pi_{1}\left(\partial_{\emptyset} X\right) & \longrightarrow & \pi_{1}\left(\partial_{0} X\right) \\
\downarrow & & \downarrow \\
\pi_{1}\left(\partial_{1} X\right) & \longrightarrow & \pi_{1}(X)
\end{array}\right)
$$

be the square of fundamental groups with orientations. The structure sets above fit 
into the following commutative diagram of exact sequences (cf. $[\mathbf{8}, \mathbf{9}]$ and $[\mathbf{1 0}])$ :

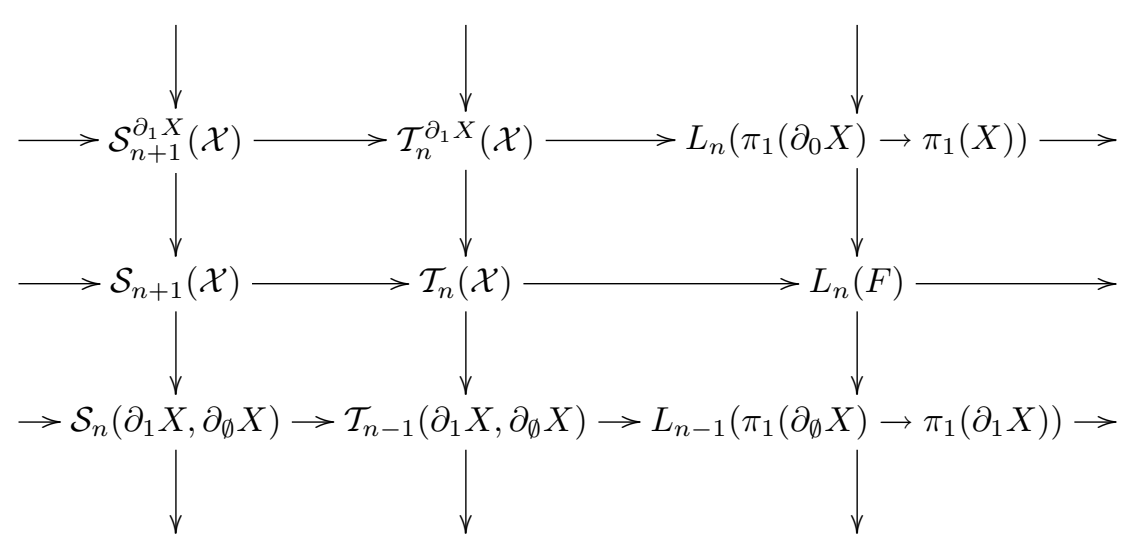

where

$$
\begin{gathered}
\mathcal{T}_{n}(\mathcal{X}) \cong \mathcal{T}(\mathcal{X}), \quad \mathcal{S}_{n+1}(\mathcal{X}) \cong \mathcal{S}(\mathcal{X}), \\
\mathcal{T}_{n}^{\partial_{1} X}(\mathcal{X}) \cong \mathcal{T}^{\partial_{1} X}(\mathcal{X}), \quad \mathcal{S}_{n+1}^{\partial_{1} X}(\mathcal{X}) \cong \mathcal{S}^{\partial_{1} X}(\mathcal{X}), \\
\mathcal{S}_{n}\left(\partial_{1} X, \partial_{\emptyset} X\right) \cong \mathcal{S}\left(\partial_{1} X, \partial_{\emptyset} X\right), \quad \text { and } \quad \mathcal{T}_{n-1}\left(\partial_{1} X, \partial_{\emptyset} X\right) \cong \mathcal{T}\left(\partial_{1} X, \partial_{\emptyset} X\right) .
\end{gathered}
$$

Wall pointed out the existence of mixed structures (cf. pp. 115 and 116 in [10]) of $n$-ads. Now, similar to the definition of the $\mathcal{T} \mathcal{S}$-structure on $(X, \partial X)$ in Section 2, we can define a $\mathcal{T} \mathcal{S}$-structure on $\left(\mathcal{X} ; \partial_{1} X\right)$ in $(67)$. This is the $\mathcal{T}$-structure on $\mathcal{X}$ whose restriction to $\left(\partial_{1} X, \partial_{\emptyset} X\right)$ is the $\mathcal{S}$-structure.

Definition 5.6. Let $f: \mathcal{M} \rightarrow \mathcal{X}$ be a $t$-triangulation

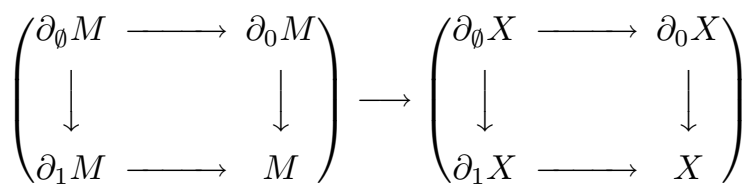

of a triad in (67) such that the restriction

$$
\left.f\right|_{\left(\partial_{1} M, \partial_{\emptyset} M\right)}:\left(\partial_{1} M, \partial_{\emptyset} M\right) \rightarrow\left(\partial_{1} X, \partial_{\emptyset} X\right)
$$

is an $s$-triangulation. Two such maps $f_{i}: \mathcal{M}_{i} \rightarrow \mathcal{X}, i=0,1$, are concordant if there exists a $t$-triangulation of the 4 -ad $\mathcal{X} \times I$ (cf. p. 111 in [10])

$$
F:\left(W ; W_{0} \cup W_{1}, V_{0}, V_{1}\right) \rightarrow\left(X \times I ; X \times\{0\} \cup X \times\{1\}, \partial_{0} X \times I, \partial_{1} X \times I\right)
$$

such that

$$
\partial V_{i}=\partial_{i} M_{0} \cup \partial_{i} M_{1} \cup S
$$

and $\left.F\right|_{V_{1}}$ is an $s$-triangulation

$$
\left(V_{1} ; \partial_{1} M_{0}, \partial_{1} M_{1}, S\right) \rightarrow\left(\partial_{1} X \times I ; \partial_{1} X \times\{0\}, \partial_{1} X \times\{1\}, \partial_{\emptyset} X \times I\right) .
$$

The set of equivalence classes of such maps is denoted by $\mathcal{T} \mathcal{S}\left(\mathcal{X} ; \partial_{1} X\right)$. 
Diagram (69) is realized on the spectrum level by the following homotopy commutative diagram of cofibrations

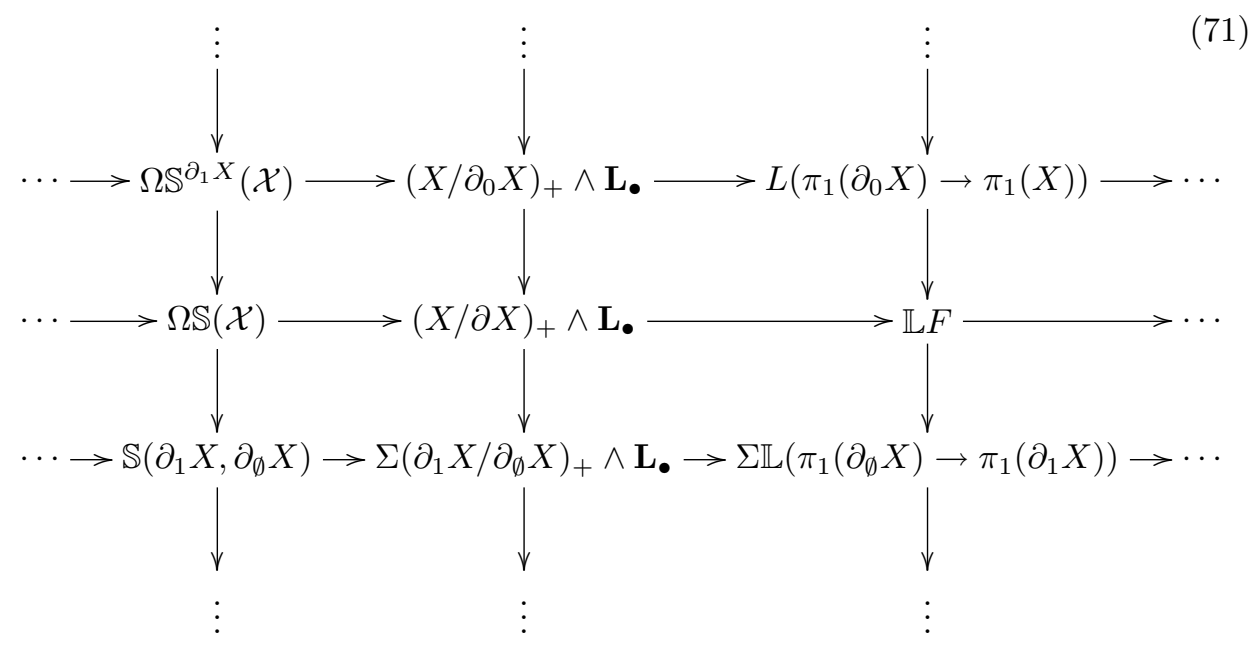

which is similar to digaram (25).

Theorem 5.7. There exists a spectrum $\mathbb{T} \mathbb{S}\left(\mathcal{X} ; \partial_{1} X\right)$ with the homotopy groups

$$
\pi_{i}\left(\mathbb{T S}\left(\mathcal{X} ; \partial_{1} X\right)\right)=\mathcal{T} \mathcal{S}_{i}\left(\mathcal{X} ; \partial_{1} X\right)
$$

fitting into the cofibrations

$$
\begin{aligned}
& \Omega \mathbb{T S}\left(\mathcal{X} ; \partial_{1} X\right) \longrightarrow \mathbb{L}\left(\pi_{1}\left(\partial_{0} X\right) \rightarrow \pi_{1}(X)\right) \longrightarrow \quad \mathbb{S}(\mathcal{X}), \\
& \Omega \mathbb{T S}\left(\mathcal{X} ; \partial_{1} X\right) \longrightarrow(X / \partial X)_{+} \wedge \mathbf{L} \bullet \quad \longrightarrow \Sigma \mathbb{L}\left(\pi_{1}\left(\partial_{\emptyset} X\right) \rightarrow \pi_{1}\left(\partial_{1} X\right)\right), \\
& \Omega \mathbb{T S}\left(\mathcal{X} ; \partial_{1} X\right) \longrightarrow \mathbb{S}\left(\partial_{1} X, \partial_{\emptyset} X\right) \longrightarrow \Sigma\left(\left(X / \partial_{0} X\right)_{+} \wedge \mathbf{L}_{\bullet}\right),
\end{aligned}
$$

such that $\mathcal{T} \mathcal{S}\left(\mathcal{X} ; \partial_{1} X\right)=\mathcal{T} \mathcal{S}_{n}\left(\mathcal{X} ; \partial_{1} X\right)$.

Proof. We can use diagram (71), similar to diagram (25), to define a spectrum $\mathbb{T S}\left(\mathcal{X} ; \partial_{1} X\right)$ fitting into the cofibrations (72). From this the result follows using standard line of argument (cf. [4] and [10]).

Remark 5.8. Similar to the construction of diagrams (31)-(33), it is now easy to write down braids of exact sequences which connect the groups $\mathcal{T} \mathcal{S}_{i}\left(\mathcal{X} ; \partial_{1} X\right)$ with others groups from diagram (69).

Example 5.9. Several structures from (52)-(56) coincide with the structures on the corresponding manifold triads. For example, for a pair $Q$ in (1) consider the manifold triad

$$
\mathcal{Z}=\left(X \backslash Y ; \partial_{0}(X \backslash Y), \partial_{1}(X \backslash Y)\right)=(X \backslash Y ; \partial X \backslash \partial Y, \partial U),
$$

where $\partial U$ is a tubular neighborhood of $Y$ in $X, X \backslash Y=\overline{X \backslash U}$, and similarly the definition we use for $\partial X \backslash \partial Y$. Now, the second structure in (52) and the second 
structure in (53) provide the structure sets which coincide with

$$
\mathcal{S}^{\partial_{1}(X \backslash Y)}(X \backslash Y ; \partial X \backslash \partial Y, \partial U)
$$

and

$$
\mathcal{T}^{\partial_{1}(X \backslash Y)}(X \backslash Y ; \partial X \backslash \partial Y, \partial U)
$$

of the triple (73), respectively.

For $Q$ in (1), consider the manifold triad

$$
\mathcal{X}=\left(X ; \partial_{0} X, \partial_{1} X\right)=\left(X ; \partial X \backslash \partial Y, U_{\partial Y}\right)
$$

where $U_{\partial Y}$ is a tubular neighborhood of $\partial Y$ in $\partial X$. The second structure in (56) is $\mathcal{S}^{\partial_{1} X}(\mathcal{X})$-structure on $\mathcal{X}$ in (74), and the third structure in (56) is the structure $\mathcal{S}(\mathcal{X})$ on $\mathcal{X}$ in (74) (cf. $[\mathbf{8}, \mathbf{9}, \mathbf{1 0}])$. The fourth structure in (53) is $\mathcal{T}^{\partial_{1} X}(\mathcal{X})$-structure on $\mathcal{X}$ in $(74)$.

Example 5.10. In the conditions of Example 5.5 for a triple in (66) we can write down the following commutative diagram (cf. [9] and [10])

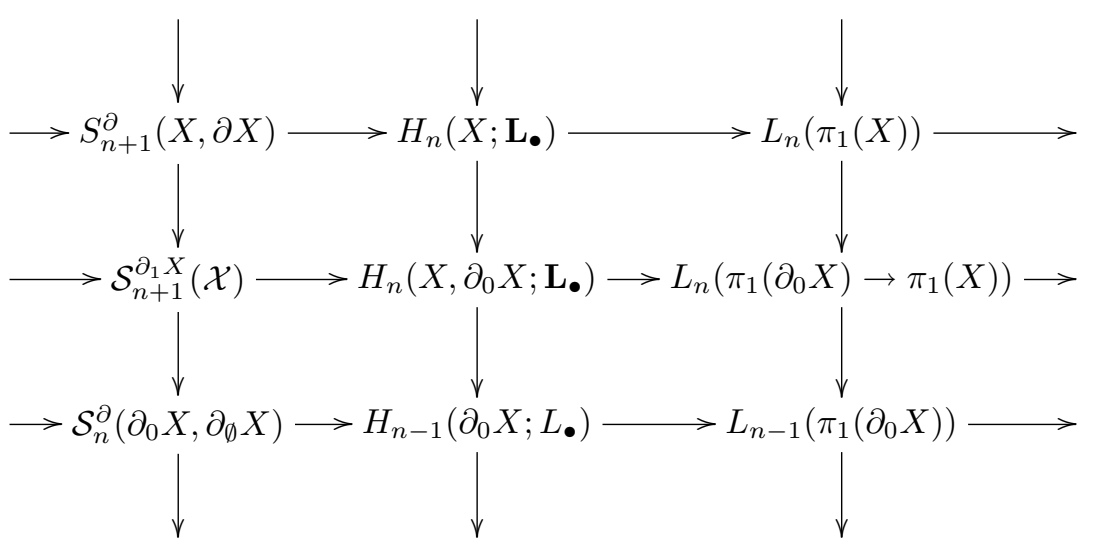

where

$$
\begin{aligned}
H_{n}\left(X ; \mathbf{L}_{\bullet}\right) & =\mathcal{T}_{n}^{\partial}(X, \partial X), \\
H_{n}\left(X, \partial_{0} X ; \mathbf{L}_{\bullet}\right) & =\mathcal{T}_{n}^{\partial_{1} X}(\mathcal{X}),
\end{aligned}
$$

and

$$
H_{n-1}\left(\partial_{0} X ; \mathbf{L}_{\bullet}\right)=\mathcal{T}_{n-1}^{\partial}\left(\partial_{0} X, \partial_{\emptyset} X\right)
$$

Diagram (75) describes connections between various structure sets and obstruction groups for the case of a manifold triple $Q$ in (66) rel $\partial_{1} X$. Similar to the consideration above, we can introduce the mixed structure set $\mathcal{T} \mathcal{S}^{\partial_{1} X}\left(\mathcal{X}, \partial_{0} X\right)$ on $Q$ rel $\partial_{1} X$. This structure set consists of the classes of concordance of $t$-triangulations of $\mathcal{X}$ rel $\partial_{1} X$ whose restrictions to $\partial_{0} X$ provide classes of concordance of $s$-triangulations rel $\partial_{\emptyset} X$. Since diagram (75) is realized on the spectrum level the introduced structure set is realized on the spectrum level by the spectrum $\mathbb{T S}^{\partial_{1} X}\left(\mathcal{X}, \partial_{0} X\right)$ with

$$
\pi_{i}\left(\mathbb{T S}^{\partial_{1} X}\left(\mathcal{X}, \partial_{0} X\right)\right)=\mathcal{T} \mathcal{S}_{i}^{\partial_{1} X}\left(\mathcal{X}, \partial_{0} X\right), \quad \mathcal{T} \mathcal{S}_{n}^{\partial_{1} X}\left(\mathcal{X}, \partial_{0} X\right) \cong \mathcal{T S}^{\partial_{1} X}\left(\mathcal{X}, \partial_{0} X\right)
$$


Since diagram (44) is realized on the spectrum level, similar to Theorem 3, we obtain the following result.

Theorem 5.11. The spectrum $\mathbb{T S}^{\partial_{1} X}\left(\mathcal{X}, \partial_{0} X\right)$ fits into the following cofibrations:

$$
\begin{aligned}
& \Omega \mathbb{T S}^{\partial_{1} X}\left(\mathcal{X}, \partial_{0} X\right) \longrightarrow \mathbb{L}\left(\pi_{1}(X)\right) \longrightarrow \mathbb{S}^{\partial_{1} X}(\mathcal{X}) \\
& \Omega \mathbb{T} \mathbb{S}^{\partial_{1} X}\left(\mathcal{X}, \partial_{0} X\right) \longrightarrow\left(X / \partial_{0} X\right)_{+} \wedge \mathbf{L}_{\bullet} \longrightarrow \Sigma \mathbb{L}\left(\pi_{1}\left(\partial_{0} X\right)\right) \\
& \Omega \operatorname{TS}^{\partial_{1} X}\left(\mathcal{X}, \partial_{0} X\right) \longrightarrow \mathbb{S}^{\partial}\left(\partial_{0} X, \partial_{\emptyset} X\right) \longrightarrow \Sigma\left(X_{+} \wedge \mathbf{L}_{\bullet}\right) \text {. }
\end{aligned}
$$

Remark 5.12. Similar to the construction of the diagrams (31)-(33), it is now easy to write down braids of exact sequences which connect the groups $\mathcal{T} \mathcal{S}_{i}^{\partial_{1} X}\left(\mathcal{X}, \partial_{0} X\right)$ with other groups from the diagram (75).

Example 5.13. The first structure in (55) is presented by the structure set

$$
\mathcal{T S}^{\partial_{1} Z}\left(\mathcal{Z}, \partial_{0} Z\right)
$$

from (73) and the last structure in (55) is presented by the structure set

$$
\mathcal{T S}^{\partial_{1} X}\left(\mathcal{X}, \partial_{0} X\right)
$$

from (74).

Example 5.14. The structure $\left[\begin{array}{l}\mathcal{S S} \\ \mathcal{S S}\end{array}\right]$ in $(52)$ is given by the classes of concordance of $s$-triangulations of $(X, \partial X)$ which are split along $Y$ and split along $\partial Y$ on the boundary (cf. [5] and [9]). This structure corresponds to the structure set

$$
\mathcal{S}(X, Y ; \partial)=\mathcal{S}(X, Y ; \partial X, \partial Y)=\mathcal{S}(X, Y, \xi ; \partial X, \partial Y, \partial \xi)
$$

described in [5].

Example 5.15. The third structure in (54) is given by the classes of concordance of $t$-triangulations of $(X, \partial X)$ whose restrictions to the boundary provides classes of concordance of $s$-triangulations $\partial X$ which are split along $\partial Y$. Denote the corresponding structure set by $\mathcal{T} \mathcal{S}(X ; \partial X, \partial Y)$. This structure set is realized by the spectrum $\mathbb{T S}(X ; \partial X, \partial Y)$ with

$$
\begin{aligned}
\pi_{i}(\mathbb{T S}(X ; \partial X, \partial Y)) & =\mathcal{T} \mathcal{S}_{i}(X ; \partial X, \partial Y) \\
\mathcal{T} \mathcal{S}_{n}(X ; \partial X, \partial Y) & =\mathcal{T} \mathcal{S}(X ; \partial X, \partial Y)
\end{aligned}
$$

(cf. $[\mathbf{5}]$ and $[\mathbf{9}]$ ).

Consider the commutative diagram of structures on $Q$ in (1)

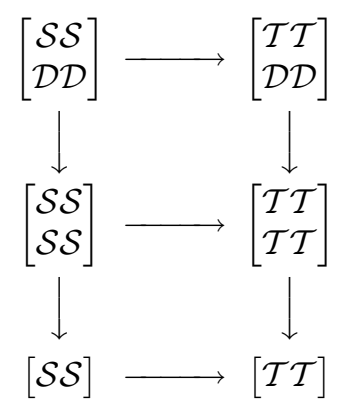


where all the maps in the upper square and the bottom horizontal map are the maps of 'weakening of the structure', and the vertical maps in the bottom square are given by the restriction of the structure to the submanifold. Diagram (77) induces the following commutative diagram of structure sets (cf. $[\mathbf{5}, \mathbf{8}, \mathbf{9}]$ and $[\mathbf{1 0}]$ )

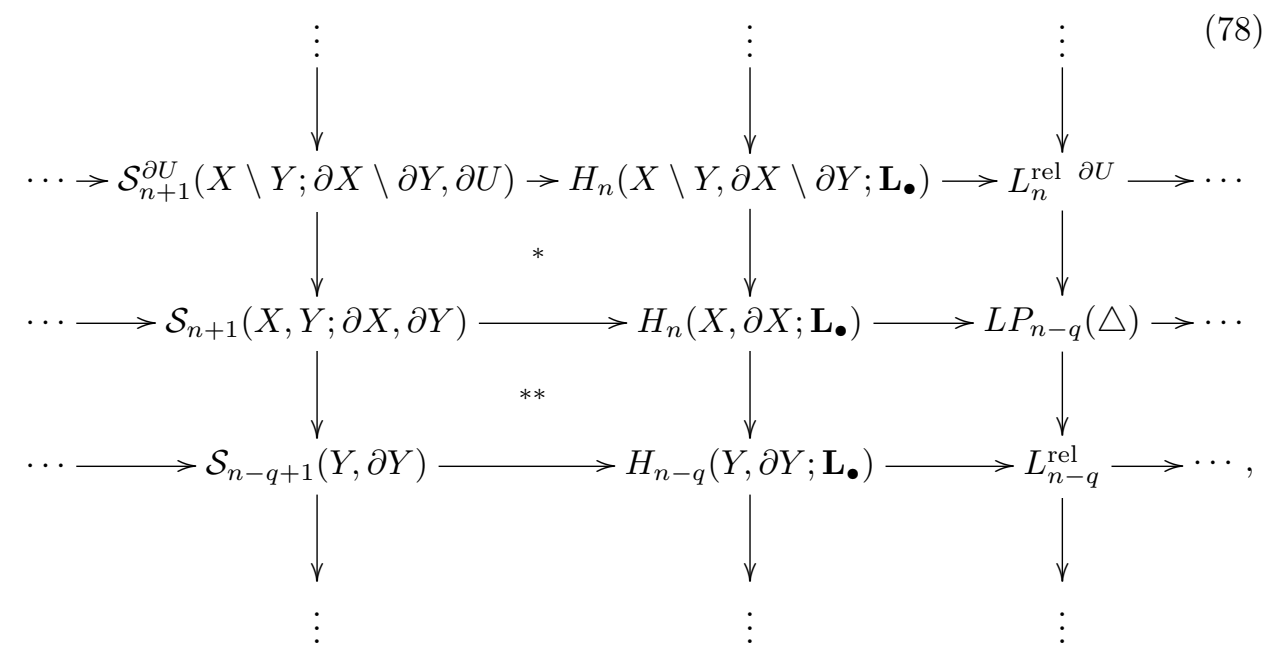

where the first row is the surgery exact sequence of the triple in (73) for the case rel $\partial U$ with $L_{*}^{\text {rel }} \partial U=L_{*}\left(\pi_{1}(\partial X \backslash \partial Y) \rightarrow \pi_{1}(X \backslash Y)\right)$ and $L_{*}^{\text {rel }}=L_{*}\left(\pi_{1}(\partial Y) \rightarrow \pi_{1}(Y)\right)$. The second row of (78) follows from diagram 3.34 of [5]. The bottom row in (78) is the surgery exact sequence of the manifold pair $(Y, \partial Y)$.

Diagram (78) is realized on the spectrum level and we have the following isomorphisms:

$$
\begin{aligned}
\mathcal{S}^{\partial U}(X \backslash Y ; \partial X \backslash \partial Y, \partial U) & \cong \mathcal{S}_{n+1}^{\partial U}(X \backslash Y ; \partial X \backslash \partial Y, \partial U), \\
\mathcal{S}(X, Y ; \partial X, \partial Y) & \cong \mathcal{S}_{n+1}(X, Y ; \partial X, \partial Y), \\
\mathcal{T}^{\partial U}(X \backslash Y ; \partial X \backslash \partial Y, \partial U) & \cong H_{n}\left(X \backslash Y, \partial X \backslash \partial Y ; \mathbf{L}_{\bullet}\right) .
\end{aligned}
$$

Example 5.16. Using the same line of argument as above, we can define a structure set $\mathcal{N S}^{\text {rel }}(X, Y ; \partial X, \partial Y)$, which consists of the classes of $\mathcal{N S}$-structures that give $\mathcal{N S}$ structures on the boundary $(\partial X, \partial Y)$. This case corresponds to the $\left[\begin{array}{l}\mathcal{T} \mathcal{T} \\ \mathcal{S} \mathcal{S}\end{array}\right]$-structure from the list in (54) on the manifold $Q$ in (1).

Theorem 5.17. There exists a spectrum $\mathbb{N} \mathbb{S}^{\mathrm{rel}}(X, Y ; \partial X, \partial Y)$ with homotopy groups

$$
\begin{aligned}
\pi_{i}\left(\mathbb{N S}^{\mathrm{rel}}(X, Y ; \partial X, \partial Y)\right) & =\mathcal{N S}_{i}^{\mathrm{rel}}(X, Y ; \partial X, \partial Y), \\
\mathcal{N S}^{\mathrm{rel}}(X, Y ; \partial X, \partial Y) & =\mathcal{N S}_{n}^{\mathrm{rel}}(X, Y ; \partial X, \partial Y) .
\end{aligned}
$$

This spectrum fits into the cofibrations

$$
\begin{gathered}
\mathbb{N S}^{r e l}(X, Y ; \partial X, \partial Y) \longrightarrow \mathbb{L}\left(\pi_{1}(\partial X \backslash \partial Y) \rightarrow \pi_{1}(X \backslash Y)\right) \longrightarrow \mathbb{S}(X, Y ; \partial), \\
\mathbb{N S}^{r e l}(X, Y ; \partial X, \partial Y) \longrightarrow(X / \partial X)_{+} \wedge \mathbf{L} \bullet \longrightarrow \Sigma^{q} \mathbb{L}\left(\pi_{1}(\partial Y) \rightarrow \pi_{1}(Y)\right), \\
\mathbb{N S}^{r e l}(X, Y ; \partial X, \partial Y) \longrightarrow \Sigma^{q-1} \mathbb{S}(Y, \partial Y) \longrightarrow \Sigma^{1}\left([(X \backslash Y) /(\partial X \backslash Y)]_{+} \wedge \mathbf{L}_{\bullet}\right) .
\end{gathered}
$$


Proof. The standard line of argument as before provides this result (cf. also $[\mathbf{2}, \mathbf{5}, \mathbf{9}]$ and $[\mathbf{1 0}])$.

Remark 5.18. Similarly to the constructions above, it is now easy to write down braids of exact sequences which connect the groups $\mathcal{N} \mathcal{S}_{i}^{\text {rel }}(X, Y ; \partial X, \partial Y)$ with other groups from diagram (78).

Remark 5.19. Note that using the standard argument of this section it is possible to define other structure sets for the structures

$$
\left[\begin{array}{l}
\mathcal{S S} \\
\mathcal{S D}
\end{array}\right],\left[\begin{array}{c}
\mathcal{T} \mathcal{S} \\
\mathcal{S S}
\end{array}\right],\left[\begin{array}{l}
\mathcal{T} \mathcal{S} \\
\mathcal{S D}
\end{array}\right]\left[\begin{array}{l}
\mathcal{T} \mathcal{T} \\
\mathcal{T} \mathcal{S}
\end{array}\right],\left[\begin{array}{l}
\mathcal{T} \mathcal{T} \\
\mathcal{S D}
\end{array}\right]
$$

from (52)-(55) which are not defined above.

\section{On the surgery obstruction groups}

The constructions of spectra for various structure sets provides also the natural maps of spectra which correspond to the maps of 'weakening of the structure' for structure sets. The cofibres of such maps are the surgery spectra for the corresponding obstruction groups. Below we consider several examples.

Example 6.1. For a closed manifold $X$ we have only one map (3) of 'weakening of the structure'. This map induces the cofibration

$$
\Omega \mathbb{S}(X) \rightarrow X_{+} \wedge \mathbf{L}_{\bullet} \rightarrow \mathbb{L}\left(\pi_{1}(X)\right)
$$

which is equivalent to the cofibration (21).

Example 6.2. For a manifold with boundary $(X, \partial X)$ we have the diagram (9) for structures. Thus we obtain the following maps of 'weakening of the structure' and corresponding cofibrations:

$$
\begin{aligned}
& \mathcal{S D} \rightarrow \mathcal{T} \mathcal{D}, \\
& \Omega \mathbb{S}^{\partial}(X, \partial X) \rightarrow X_{+} \wedge \mathbf{L} \bullet \rightarrow \mathbb{L}\left(\pi_{1}(X)\right),
\end{aligned}
$$

which coincide with the upper row in (25);

$$
\begin{gathered}
\mathcal{S S} \rightarrow \mathcal{T} \mathcal{T}, \\
\Omega \mathbb{S}(X, \partial X) \rightarrow(X / \partial X)_{+} \wedge \mathbf{L}_{\bullet} \rightarrow \mathbb{L}^{\text {rel }},
\end{gathered}
$$

which coincide with the middle row in $(25)$;

$$
\begin{gathered}
\mathcal{T S} \rightarrow \mathcal{T} \mathcal{T}, \\
\Omega \mathbb{T S}(X, \partial X) \rightarrow(X / \partial X)_{+} \wedge \mathbf{L}_{\bullet} \rightarrow \Sigma \mathbb{L}\left(\pi_{1}(\partial X)\right),
\end{gathered}
$$

which coincide with the middle row in (30);

$$
\begin{aligned}
\mathcal{T} \mathcal{D} & \rightarrow \mathcal{T} \mathcal{T}, \\
X_{+} \wedge \mathbf{L}_{\bullet} \rightarrow(X / \partial X)_{+} & \wedge \mathbf{L}_{\bullet} \rightarrow \Sigma\left(\left(\partial X_{+}\right) \wedge \mathbf{L}_{\bullet}\right),
\end{aligned}
$$

which coincide with the middle column in (25);

$$
\begin{gathered}
\mathcal{S D} \rightarrow \mathcal{S S} \\
\mathbb{S}^{\partial}(X, \partial X) \rightarrow \mathbb{S}(X, \partial X) \rightarrow \Sigma \mathbb{S}(\partial X),
\end{gathered}
$$


which coincide with the left column in (25);

$$
\begin{aligned}
& \mathcal{T D} \rightarrow \mathcal{T S}, \\
X_{+} \wedge \mathbf{L}_{\bullet} \rightarrow & \Omega \mathbb{T S}(X, \partial X) \rightarrow \mathbb{S}(\partial X),
\end{aligned}
$$

which is equivalent to the bottom row in $(30)$;

$$
\begin{gathered}
\mathcal{S S} \rightarrow \mathcal{T S}, \\
\mathbb{S}(X, \partial X) \rightarrow \mathbb{T S}(X, \partial X) \rightarrow \Sigma \mathbb{L}\left(\pi_{1}(X)\right)
\end{gathered}
$$

which is equivalent to the upper row in (30). For the maps

$$
\begin{aligned}
& \mathcal{S D} \rightarrow \mathcal{T} \mathcal{S}, \\
& \mathcal{S D} \rightarrow \mathcal{T} \mathcal{T},
\end{aligned}
$$

which follow from (9) the obstruction groups have not been introduced so far. Now it is an easy exercise to introduce the surgery spectra for the corresponding obstruction groups and commutative diagrams and braids of exact sequences, similar to above.

For the case of a manifold pair $(X, Y)$ we can write down the maps of 'weakening of the structure' and the corresponding cofibrations, similar to Example 6.2. Diagram (15) on the spectrum level provides the maps of spectra for structure sets, and as cofibres of the maps we shall obtain spectra for corresponding obstruction groups. In this case we can also write additional cofibrations of spectra for structure sets (with cofibres which are spectra for corresponding obstruction groups) which provides commutative diagram of structures (17).

For a manifold pair with boundary we have a great number of maps of 'weakening of the structure', but now it is sufficiently easy to construct spectra for corresponding obstruction groups and describe theirs properties. Many of such examples are given in Section 5 .

Example 6.3. For a manifold pair with boundary (1), there is the following map of 'weakening of the structure':

$$
\left[\begin{array}{l}
\mathcal{S S} \\
\mathcal{S S}
\end{array}\right] \longrightarrow\left[\begin{array}{c}
\mathcal{T} \mathcal{S} \\
\mathcal{T} \mathcal{T}
\end{array}\right]
$$

which provides the following cofibration (cf. Theorem 2 of [5]):

$$
\mathbb{S}(X, Y, \partial) \rightarrow \mathbb{T S}(X, \partial X) \longrightarrow \Sigma^{q+1} \mathbb{L} \mathbb{P S}(\Delta)
$$

where the groups $\operatorname{LPS}_{i}(\Delta)=\pi_{i}(\mathbb{L} \mathbb{P S}(\Delta))$ are the corresponding obstruction groups.

\section{References}

[1] A. Bak and Yu.V. Muranov, Splitting along submanifolds and L-spectra, Contemporary Mathematics and Applications: Topology, Calculus, and Related Questions, Academy of Sciences of Georgia, Inst. of Cybernetics, Tbilisi 1 (2003), $3-18$.

[2] A. Bak and Yu.V. Muranov, Normal invariants of pairs of manifolds and signature mappings, Mat. Sb. 197 (2006), no. 6, 3-24; English transl., Sb. Math. 197 (2006), no. 5-6, 791-811. 
[3] A. Cavicchioli, Yu.V. Muranov, and F. Spaggiari, Relative groups in surgery theory, Bull. Belg. Math. Soc. Simon Stevin 12 (2005), 109-135.

[4] A. Cavicchioli, Yu.V. Muranov, and F. Spaggiari, Mixed structures on a manifold with boundary, Glasgow Math. J. 48 (2006), no. 1, 125-143.

[5] M. Cencelj, Yu.V. Muranov, and D. Repovš, On the splitting problem for manifold pairs with boundaries, Abh. Math. Sem. Univ. Hamburg 76 (2006), 35-55.

[6] I. Hambleton, A.A. Ranicki, and L. Taylor, Round L-theory, J. Pure Appl. Algebra 47 (1987), 131-154.

[7] Yu.V. Muranov, Obstruction groups to splitting, and quadratic extensions of antistructures, Izvestiya RAN: Ser. Mat. 59 (1995), no. 6, 107-132; English transl., Izvestiya Math. 59 (1995), no. 6, 1207-1232.

[8] A.A. Ranicki, The Total Surgery Obstruction, Lecture Notes in Math. 763 (1979), 275-316.

[9] A.A. Ranicki, Exact sequences in the algebraic theory of surgery, Math. Notes 26, Princeton Univ. Press, Princeton, N.J., 1981.

[10] C.T.C. Wall, Surgery on compact manifolds, Second Edition, Edited by A.A. Ranicki, Amer. Math. Soc., Providence, R.I., 1999.

[11] S. Weinberger, The topological classification of stratified spaces, Chicago Lectures in Math., The University of Chicago Press, Chicago, 1994.

Matija Cencelj matija.cencelj@guest.arnes.si

Institute of Mathematics, Physics and Mechanics, and Faculty of Education, University of Ljubljana, Jadranska 19, SI-1000 Ljubljana, Slovenia

Yuri V. Muranov ymuranov@mail.ru

Physical Department, Vitebsk State University, Moskovskii pr. 33, 210026 Vitebsk, Belarus

Dušan Repovš dusan.repovs@guest.arnes.si

Faculty of Mathematics and Physics, and Faculty of Education, University of Ljubljana, Jadranska 19, SI-1000 Ljubljana, Slovenia 